\title{
Dynamic Modeling of a 2-RPU+2-UPS Hybrid Manipulator for Machining Application
}

\author{
Ruiqin $\mathrm{Li}^{1}$ Shusen Wang ${ }^{1}$ Dabao Fan ${ }^{1}$ Yuting Du ${ }^{1}$ Shaoping Bai ${ }^{2}$ \\ ${ }^{1}$ School of Mechanical Engineering, North University of China, Taiyuan, China. E-mail: liruiqin@nuc.edu.cn \\ ${ }^{2}$ Department of Mechanical and Manufacturing Engineering, Aalborg University, Aalborg, Denmark. E-mail: \\ shb@m-tech.aau.dk
}

\begin{abstract}
This paper presents a novel 5-DOF gantry hybrid machine tool, designed with a 2-RPU+2-UPS parallel mechanism for $3 \mathrm{~T} 2 \mathrm{R}$ motion. The 2-RPU+2-UPS parallel mechanism is connected to a long linear guide to realize 5-axis machining. A dynamic model is developed for this parallel-serial hybrid system. Screw theory is adopted to establish the kinematic equations of the system, upon which the dynamics model is developed by utilizing the principle of virtual work. A numerical example for processing slender structural parts is included to show the validity of the analytical dynamic model developed.
\end{abstract}

Keywords: gantry hybrid machine tool, 2-RPU+2-UPS, kinematics and dynamics modeling, large-scale structural part machining

\section{Introduction}

In a hybrid configuration, a mechanism combines both series and parallel kinematic chains. Hybrid mechanisms have attracted significant attention from academia and industry due to their high stiffness, high precision, large workspace, flexibility, and other performance advantages (Merlet (2002) and Gao and Zhang (2015)).

Many different hybrid mechanisms have been proposed. A hybrid mechanism, PARASURG-9M, proposed by Pisla et al. (2013), was used for minimally invasive surgery. It is composed of the 5-DOF series positioning module PARASURG-5M and 4-DOF parallel module PARASIM (Vaida et al. (2010)). Three hybrid mechanisms of 6 -DOF constructed by serially connecting 2 parallel mechanisms were presented by Hu et al. (2011), Hu et al. (2012), Hu and Yu (2015), which include 2(SP+SPR+SPU), (3-RPS $)+(3-\mathrm{SPR})$, and (UPR+RPS+UPS)+(3-UPS/UP). Hereinafter, U, $\mathrm{P}, \mathrm{R}, \mathrm{S}$ stand for universal/Hooke, prismatic, revolute and spherical joints. A 2-DOF hybrid mechanism used for horizontal machine tools was presented by Jiang et al. (2015). It is composed of a 2-DOF redundant driving planar parallel mechanism with a 2-DOF mobile platform. Liang and Ceccarelli (2012) proposed a hybrid mechanism for a waist-trunk system for a humanoid robot. The hybrid mechanism is constructed with chains in series of a 3 -DOF 3 -SPS $+\mathrm{S}$ parallel mechanism and a 6-DOF 6-SPS parallel mechanism. Huang et al. (2010) developed a 4-DOF hybrid kinematic machine composed of a 2-DOF parallel mechanism combined with a 2-DOF rotating head. This hybrid machine is configured for a robot cell moving along a long track for aircraft wing box assemblies. Gallardo-Alvarado et al. (2012) proposed a 6-DOF 3PPS+3-RPS hybrid mechanism. This hybrid mechanism has a decoupled topology feature. Huang et al. (2011) developed a configuration of a 3-P(4R)S-XY hybrid machine tool and derived the error model and error kinematics. Lu et al. (2014) proposed a 6-DOF 3UPS parallel manipulator with multiple fingers; their manipulator has three fingers installed on a moving platform, which decreases interference and enlarged its 
position and orientation workspace.

In addition, Assal (2015) designed a planar parallel manipulator with high orientation ability for a hybrid machine tool. Wu et al. (2015b) established an effective dynamic model that took into consideration the deformation of the flexible link of the heavy duty parallel manipulator.

Giving many hybrid manipulators proposed, however, the literatures are mainly limited to the kinematics modeling, with very few on dynamics modeling. The rigid-body dynamics model of the 5-DOF Gantry-Tau parallel kinematic machine was verified with experiment by Lyzell and Hovland (2007). A general method to calculate the inverse and direct dynamic models of parallel robots with closed expression is presented by Khalil and Ibrahim (2007). The dynamic performance of a new 5-DOF hybrid machine tool composed of a 3-DOF parallel manipulator combined with a 2-DOF feed worktable was analyzed by Li et al. (2010). The stiffness of a 5-DOF hybrid machine tool composed of 2 parallel mechanisms was analyzed by Lian et al. (2015).

This work is focused on the dynamic modeling of hybrid manipulators. While dynamic approaches, including the Lagrange method (Liu and Yu (2008), Wu et al. (2014)), Newton-Euler method (Jalón and Bayo (1994), Zhang et al. (2009)), Kane method (Cheng and Shan (2012)), principle of virtual work (Sokolov and Xirouchakis (2007)) and (Zhao et al. (2009)), and screw theory (Gallardo-Alvarado et al. (2008)) are commonly used and applied in manipulator dynamics (Horn and Linge (1995), Wu and Bai (2016)), this work adopts the principle of virtual work, under the consideration to eliminate the internal forces and effectively reduce the computational complexity.

The motivation behind the modeling work pertains to the design of a novel 5-DOF Gantry Hybrid Machine Tool (GHMT for short) to machine relatively large and slender structural parts with complex curved surfaces that are often used in railway carriages, aircraft wings, and wind turbine blades. The 5-DOF GHMT includes a novel 2-RPU+2-UPS parallel mechanism with 2 translations and 2 rotations. By connecting it to the slide guide in a series, the mechanism can have translations along 3 axes and yaw and pitch rotations. Not only can this hybrid tool machine process complex slender structural parts, but it provides a large workspace and better control and kinematic performance than existing machine tools.

The paper is organized as follows. The configuration of the GHMT is explained and the driving selection is described in Section 2. The kinematic model of the GHMT is established in Section 3. The dynamic model using the principle of virtual work is established in Section 4. Section 5 describes the validation of the model, followed by Section 6 with a numerical example for the driving force of the GHMT when it is used for a large slender structural part. The work is concluded in Section 7.

\section{Model of the 5-DOF GHMT}

\subsection{Configuration of the 5-DOF GHMT}

A CAD model of the 5-DOF GHMT is shown in Figure 1. Its kinematic model is presented in Figure 2.

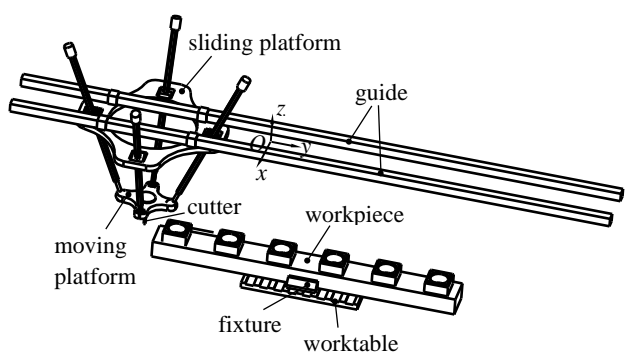

Figure 1: CAD model of the 5-DOF GHMT

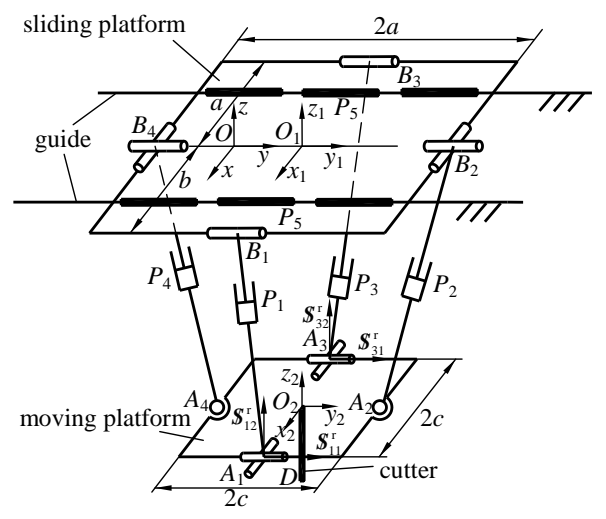

Figure 2: The schematic diagram of the 5-DOF GHMT

The 2-RPU+2-UPS parallel mechanism is comprised of a sliding platform, a moving platform (MP), two identical RPU limbs, and two identical UPS limbs. One end of the RPU limb connects the sliding platform with the revolute pair $\mathrm{R}$, and the other end connects the MP with universal joint $U$. In the two universal joints of the RPU limbs, the two axes of the first revolute pairs are collinear and the two axes of the second revolute pairs are parallel to the axis of the sliding pair. One end of the UPS limb connects the sliding platform with Hooke joint $\mathrm{U}$, and the other end connects the MP with spherical pair S. The parallel mechanism is connected to the linear guide by two sliding pairs $\mathrm{P}_{5}$. 
In Figure 2, the distances between the hinge points $B_{2}, B_{3}$, and $B_{4}$, and the sliding platform center $O_{1}$ are equal, marked as $a$. The distance between point $B_{1}$ and $O_{1}$ is marked as $b$. Four hinge points $A_{i}(i=1,2$, $3,4)$ on the MP are evenly distributed in the square with a side length of $2 c$. The length of each limb is equal to $l_{i}(i=1,2,3,4)$.

\subsection{Coordinate Systems of the 5-DOF GHMT}

Referring to Figure 2, there are three coordinate systems. The fixed coordinate system $O-x y z$, marked as $\{O\}$, is connected to the two parallel linear guides. The coordinate origin $O$ is located at the center of the two guides. The $y$-axis is parallel to the moving direction of the guides and passes through points $B_{2}$ and $B_{4}$. The $x$-axis is perpendicular to the guides.

The sliding coordinate system $O_{1}-x_{1} y_{1} z_{1}$, marked as $\left\{O_{1}\right\}$, is connected to the sliding platform. The coordinate origin $O_{1}$ is located at the center of two guides and is the midpoint between $B_{2}$ and $B_{4}$. The $y_{1}$-axis is parallel to the moving direction of the guide and passes through points $B_{2}$ and $B_{4}$. The $x_{1}$-axis passes through points $B_{1}$ and $B_{3}$.

The moving coordinate system $\mathrm{O}_{2}-x_{2} y_{2} z_{2}$, marked as $\left\{\mathrm{O}_{2}\right\}$, is connected to the MP. The coordinate origin $\mathrm{O}_{2}$ is located at the center of the MP. The $x_{2}$-axis passes through points $A_{1}$ and $A_{3}$. The $y_{2}$-axis passes through points $A_{2}$ and $A_{4}$.

The cutter axis is always aligned with the $z_{2}$-axis.

\subsection{DOF Analysis of the 5-DOF GHMT}

As shown in Figure 2, each UPS limb does not apply any constraint on the MP, while each RPU limb produces 2 constraint force screws, $\boldsymbol{\$}_{i 1}^{\mathrm{r}}$ and $\boldsymbol{\$}_{i 2}^{\mathrm{r}}(i=1,3)$, on the MP. Here, $\boldsymbol{\$}_{i 1}^{\mathrm{r}}$ is a constraint linear vector, and it is coaxial with the corresponding limb universal pair and parallel to the axis of revolute pair, i.e., parallel to the $y_{2}$ axis. $\$_{i 2}^{\mathrm{r}}$ is the constraint couple, and it is perpendicular to all shafts in the limbs, so it is perpendicular to the Hooke hinge (i.e., parallel to the $z_{2}$-axis).

Two constraint linear vectors show linear dependence, and can be expressed by the linear vector basis of the force. Two constraint couples also show linear dependence and can be expressed by the couple basis. Their expressions are as follows:

$$
\begin{aligned}
& \boldsymbol{\$}_{1}^{\mathrm{r}^{\prime}}=\left(\begin{array}{lllll}
0 & 1 & 0 ; 0 & 0 & 0
\end{array}\right) \\
& \boldsymbol{\$}_{2}^{\mathrm{r}^{\prime}}=\left(\begin{array}{lllll}
0 & 0 & 0 ; 0 & 0 & 1
\end{array}\right)
\end{aligned}
$$

According to the relationship between the kinematic screw and constraint screw in screw theory, the reciprocal screw of 2 force screws, $\$_{1}^{\mathrm{r}^{\prime}}$ and $\$_{2}^{\mathrm{r}^{\prime}}$, are 4 irrelevant kinematic screws of the MP.

$$
\begin{aligned}
& \boldsymbol{\$}_{m 1}=\left(\begin{array}{lllll}
1 & 0 & 0 ; 0 & 0 & 0
\end{array}\right) \\
& \$_{m 2}=\left(\begin{array}{lllll}
0 & 1 & 0 ; 0 & 0 & 0
\end{array}\right) \\
& \boldsymbol{\$}_{m 3}=\left(\begin{array}{lllll}
0 & 0 & 0 ; 1 & 0 & 0
\end{array}\right) \\
& \boldsymbol{\$}_{m 4}=\left(\begin{array}{lllll}
0 & 0 & 0 ; 0 & 0 & 1
\end{array}\right)
\end{aligned}
$$

These four kinematic screws reflect the unrestrained motion of two force screws, $\boldsymbol{\$}_{1}^{\mathrm{r}^{\prime}}$ and $\boldsymbol{\$}_{2}^{\mathrm{r}^{\prime}}$, relative to a $\mathrm{MP}$, thus the MP of the 2-RPU+2-UPS parallel mechanism has 4 DOFs relative to the sliding platform: the rotation around the $x_{1}$-axis and $y_{1}$-axis and translation along the $x_{1}$-axis and $z_{1}$-axis. Connecting the parallel mechanism to the guide by sliding pair $\mathrm{P}_{5}$ in a series can achieve translation along the $y$ axis. This allows for the realization of the 5 DOF movements of the hybrid machine tool.

As this GHMT has 5 DOFs, there are 5 linearly independent driving inputs. One of them is a sliding pair $\mathrm{P}_{5}$ between the sliding platform and the guide, and the other 4 driving inputs are in the 2-RPU+2-UPS parallel mechanism. To improve the performance of the GHMT, all actuations should be as close as possible to the sliding platform. Four sliding pairs, $\mathrm{P}_{1}, \mathrm{P}_{2}, \mathrm{P}_{3}$, and $\mathrm{P}_{4}$, connected to the sliding platform were selected as the driving pairs.

\section{Kinematic Model of the 5-DOF GHMT}

\subsection{The Inverse Position Analysis of the 5-DOF GHMT}

The inverse position problem of the 5-DOF GHMT is to find the displacement $s_{i}$ of the sliding pair in each limb $(i=1,2, \cdots, 5)$ for given position and orientation $\left(x_{D}, y_{D}, z_{D}, \psi, \theta, \phi\right)$ of the tooling point $D$ relative to the fixed coordinate system $\{O\}$. Moreover, $\boldsymbol{O}_{D}=\left(x_{D}, y_{D}, z_{D}\right)^{\mathrm{T}}$ is the position of tooling point $D$, and $\psi, \theta, \phi$ are Tait-Bryan angles following $Z$ $Y-X$ convention. As the mechanism has no rotational freedom around the $z$ axis, i.e., $\phi=0$, the rotation matrix $R$ is

$$
\boldsymbol{R}=\left[\begin{array}{ccc}
\mathrm{c} \theta & \mathrm{s} \theta \mathrm{s} \psi & \mathrm{s} \theta \mathrm{c} \psi \\
0 & \mathrm{c} \psi & -\mathrm{s} \psi \\
-\mathrm{s} \theta & \mathrm{c} \theta \mathrm{s} \psi & \mathrm{c} \theta \mathrm{c} \psi
\end{array}\right]
$$

Hereafter, $\mathrm{s}=\sin$ and $\mathrm{c}=\mathrm{cos}$.

As the axis of the cutter is parallel to the $z_{2}$ axis, its direction vector can be written as

$$
\boldsymbol{n}=\left(\begin{array}{lll}
\mathrm{s} \theta \mathrm{c} \psi & -\mathrm{s} \psi & \mathrm{c} \theta \mathrm{c} \psi
\end{array}\right)^{\mathrm{T}}
$$


The coordinate of the center of the MP $\mathrm{O}_{2}$, which is located at one end of the cutter shaft, is calculated by

$$
\begin{aligned}
\boldsymbol{O}_{2} & =\boldsymbol{O}_{D}+d \boldsymbol{n} \\
& =\left(\begin{array}{lll}
x_{D}+d \cdot \mathrm{s} \theta \mathrm{c} \psi & y_{D}-d \cdot \mathrm{s} \psi & z_{D}+d \cdot \mathrm{c} \theta \mathrm{c} \psi
\end{array}\right)^{\mathrm{T}}
\end{aligned}
$$

where $d$ is the length of the cutter shaft.

The coordinate of point $O_{1}$ can be expressed as

$$
\boldsymbol{O}_{1}=\left(\begin{array}{lll}
0 & y_{D}-d \cdot \mathrm{s} \psi & 0
\end{array}\right)^{\mathrm{T}}
$$

The input displacement of the active sliding pair $\mathrm{P}_{5}$ on the guide can be expressed as

$$
s_{5}=y_{D}-d \cdot \sin \psi
$$

The coordinate of the MP center relative to the sliding platform can be expressed as

$$
\begin{aligned}
{ }^{O_{1}} \boldsymbol{O}_{2} & =\boldsymbol{O}_{D}+d \boldsymbol{n} \\
& =\left(\begin{array}{lll}
x_{D}+d \cdot \mathrm{s} \theta \mathrm{c} \psi & 0 & z_{D}+d \cdot \mathrm{c} \theta \mathrm{c} \psi
\end{array}\right)^{\mathrm{T}}
\end{aligned}
$$

The position of point $B_{i}(i=1,2,3,4)$ in $\left\{O_{1}\right\}$ and point $A_{i}(i=1,2,3,4)$ in $\left\{O_{2}\right\}$ are expressed as follows, respectively:

$$
\left\{\begin{array}{l}
{ }_{O_{1}} \boldsymbol{B}_{1}=(b, 0,0)^{\mathrm{T}} \\
{ }_{O_{1}} \boldsymbol{B}_{2}=(0, a, 0)^{\mathrm{T}} \\
{ }^{O_{1}} \boldsymbol{B}_{3}=(-a, 0,0)^{\mathrm{T}} \\
{ }^{O_{1}} \boldsymbol{B}_{4}=(0,-a, 0)^{\mathrm{T}}
\end{array}, \quad\left\{\begin{array}{l}
{ }^{O_{2}} \boldsymbol{A}_{1}=(c, 0,0)^{\mathrm{T}} \\
{ }_{O_{2}} \boldsymbol{A}_{2}=(0, c, 0)^{\mathrm{T}} \\
{ }^{O_{2}} \boldsymbol{A}_{3}=(-c, 0,0)^{\mathrm{T}} \\
{ }^{O_{2}} \boldsymbol{A}_{4}=(0,-c, 0)^{\mathrm{T}}
\end{array}\right.\right.
$$

Through homogeneous coordinate transformation, the coordinate position of point $A_{i}(i=1,2,3,4)$ is transformed into $\left\{O_{1}\right\}$, and the transformation formula is

$$
{ }^{O_{1}} \boldsymbol{A}_{i}=\boldsymbol{R}^{O_{2}} \boldsymbol{A}_{i}+{ }^{O_{1}} \boldsymbol{O}_{2}
$$

Now, the vectors of the four driving parts can be expressed in $\left\{O_{1}\right\}$ :

$$
\boldsymbol{l}_{i}={ }^{O_{1}} \boldsymbol{A}_{i}-{ }^{O_{1}} \boldsymbol{B}_{i} \quad(i=1,2,3,4)
$$

Thus, the input displacement is

$$
s_{i}=l_{i}-l_{i 0} \quad(i=1,2,3,4)
$$

where $l_{i 0}$ is the initial length of the driving rod.

\subsection{The Workspace Analysis of the 5-DOF GHMT}

The range of motion of the 5-DOF GHMT can be determined geometrically.
Figure 3 shows the movement range of the MP in the $z$ direction. Extreme positions are achieved when minimum or maximum lengths of $l_{1}$ and $l_{3}$ are reached.

$$
z=z_{\max }-z_{\min }
$$

where $z_{\max }$ and $z_{\min }$ are the extreme positions of the MP. Let the MP keep horizontal, $z_{\max }$ and $z_{\min }$ are found as,

$$
\left\{\begin{array}{l}
z_{\min }=l_{3 \min } \cos \gamma_{1} \\
z_{\max }=l_{3 \max } \cos \gamma_{2} \\
\gamma_{1}=\sin ^{-1} \frac{a-c}{l_{3 \min }} \\
\gamma_{2}=\sin ^{-1} \frac{a-c}{l_{3 \max }}
\end{array}\right.
$$

where dimensions $a$ and $c$ are illustrated in Figure 2. Figure 4 shows the extreme positions of the MP along $x$ direction.

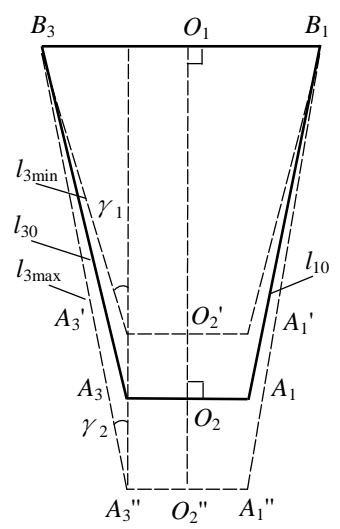

Figure 3: The movement diagram of the MP along $z$ direction, with extreme positions displayed in dashed lines

The maximum displacement of the MP along the positive $x$ axis for a given $z$ is expressed as follows:

$$
x_{+}=\sqrt{l_{m}^{2}+z^{2}}
$$

where,

$$
\begin{gathered}
l_{m}=\sqrt{(a-c)^{2}+l_{3 \max }^{2}-2(a-c) l_{3 \max } \cos \gamma_{3}} \\
\gamma_{3}=\sin ^{-1} \frac{z}{l_{3 \max }}
\end{gathered}
$$

Similarly, the movement of distance of the MP along the negative $x$ axis could be calculated by above method. 
The rotation range around $y$ axis clockwise is analyzed and the rotation extreme position of the MP around $y$ axis is showed in Figure 5.

The maximum rotation is achieved when one actuator reaches the minimum length and the other reaches the maximum. The rotation range of the $\mathrm{MP}$ around $y$ axis is calculated as follows:

$$
\phi_{\max }=\tan ^{-1} \frac{z}{a}-\cos ^{-1} \frac{c^{2}+z^{2}+a^{2}-l_{3 \min }^{2}}{2 c \sqrt{z^{2}+a^{2}}}
$$

Similarly, the rotation range around $y$ axis counterclockwise and the rotation range around $x$ axis could be calculated by the above method.

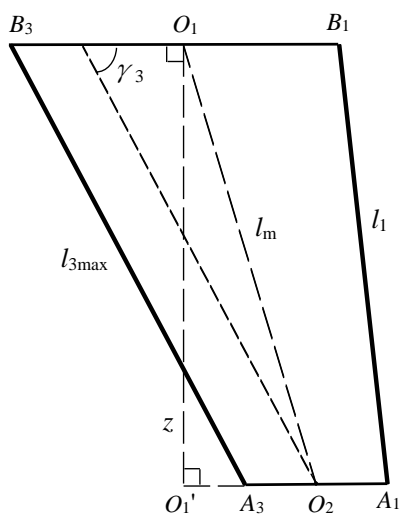

Figure 4: The extreme position of the MP along $x$ axis positive direction

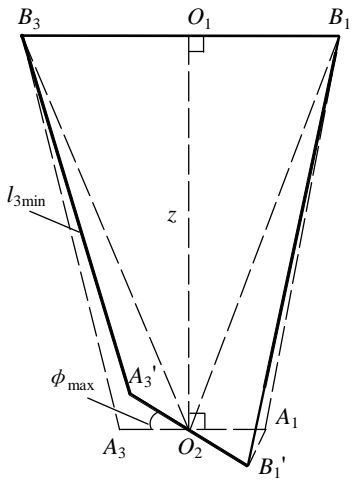

Figure 5: The rotational extreme position of the MP around $y$ axis

\subsection{The Velocity Analysis of the 5-DOF GHMT}

The vectors in Figure 6 are described in the coordinate system $\{O\}$, as shown in Table 1 .
The velocity of the end of each limb on the MP can also be expressed as

$$
\boldsymbol{v}_{i}=\boldsymbol{v}_{O 2}+\boldsymbol{\omega} \times \boldsymbol{e}_{i}
$$

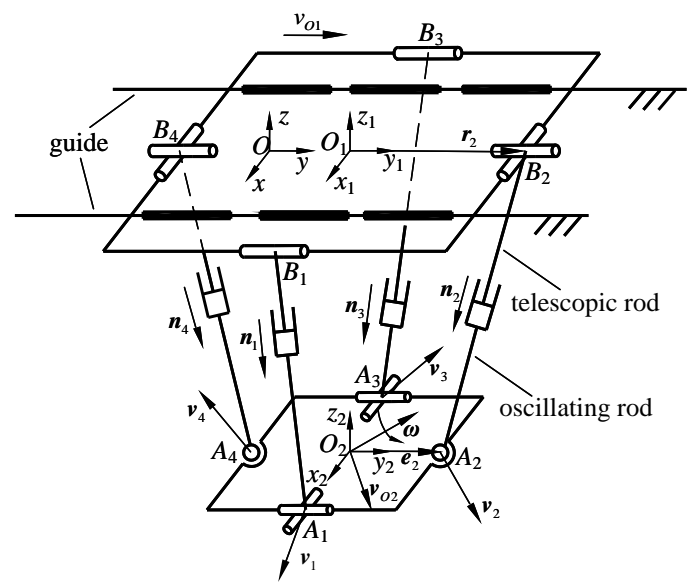

Figure 6: The velocities of the 5-DOF GHMT

Table 1: The vectors used in Figure $6(i=1,2,3,4)$

\begin{tabular}{cl}
\hline Parameters & Meaning \\
\hline$v_{\mathrm{O} 1}$ & The speed of the sliding plat- \\
& form sliding on the guide \\
$\boldsymbol{v}_{\mathrm{O} 2}$ & The velocity of the MP \\
$\boldsymbol{\omega}$ & The angular velocity vector of \\
& the MP \\
$\boldsymbol{v}_{i}$ & The velocity of point $A_{i}$ at the \\
& lower end of the driving rod \\
$\boldsymbol{n}_{i}=\frac{\left(\boldsymbol{A}_{i}-\boldsymbol{B}_{i}\right)}{\left|\boldsymbol{A}_{i}-\boldsymbol{B}_{i}\right|}$ & The unit vector along the driv- \\
$\boldsymbol{e}_{i}=\boldsymbol{A}_{i}-\boldsymbol{O}_{2}$ & The positional vector from $O_{2}$ to \\
$\boldsymbol{r}_{i}=\boldsymbol{B}_{i}-\boldsymbol{O}_{1}$ & $\begin{array}{l}A_{i} \\
\text { The positional vector from } O_{1} \text { to }\end{array}$ \\
& $B_{i}$ \\
\hline
\end{tabular}

The driving velocity $v_{l i}$ along the driving $\operatorname{rod} l_{i}(i=1$, $2,3,4)$ is expressed as follows:

$$
\begin{aligned}
v_{l i} & =\boldsymbol{v}_{i} \cdot \boldsymbol{n}_{i}=\left(\boldsymbol{v}_{O 2}+\boldsymbol{\omega} \times \boldsymbol{e}_{i}\right) \cdot \boldsymbol{n}_{i} \\
& =\left[\begin{array}{ll}
\boldsymbol{n}_{i}^{\mathrm{T}} & \left(\boldsymbol{e}_{i} \times \boldsymbol{n}_{i}\right)^{\mathrm{T}}
\end{array}\right]\left[\begin{array}{c}
\boldsymbol{v}_{O 2} \\
\boldsymbol{\omega}
\end{array}\right]
\end{aligned}
$$

Equation (21) is rewritten in matrix form as

$$
\boldsymbol{v}_{l}=\boldsymbol{J}_{l}\left[\begin{array}{c}
\boldsymbol{v}_{O 2} \\
\boldsymbol{\omega}
\end{array}\right]
$$


where

$$
\boldsymbol{J}_{l}=\left[\begin{array}{cc}
\boldsymbol{n}_{1}^{\mathrm{T}} & \left(\boldsymbol{e}_{1} \times \boldsymbol{n}_{1}\right)^{\mathrm{T}} \\
\boldsymbol{n}_{2}^{\mathrm{T}} & \left(\boldsymbol{e}_{2} \times \boldsymbol{n}_{2}\right)^{\mathrm{T}} \\
\boldsymbol{n}_{3}^{\mathrm{T}} & \left(\boldsymbol{e}_{3} \times \boldsymbol{n}_{3}\right)^{\mathrm{T}} \\
\boldsymbol{n}_{4}^{\mathrm{T}} & \left(\boldsymbol{e}_{4} \times \boldsymbol{n}_{4}\right)^{\mathrm{T}}
\end{array}\right] \quad, \quad \boldsymbol{v}_{l}=\left[\begin{array}{c}
v_{l 1} \\
v_{l 2} \\
v_{l 3} \\
v_{l 4}
\end{array}\right]
$$

where $\boldsymbol{J}_{l}$ is a $4 \times 6$ velocity Jacobian matrix. It reflects the velocity mapping relationship between the velocity of the MP and absolute driving velocity.

The position $\left(x_{\mathrm{O} 2}, y_{\mathrm{O} 2}, z_{\mathrm{O} 2}\right)$ of the $\mathrm{MP}$ center is the function of the generalized coordinates $x_{D}, y_{D}, z_{D}, \theta, \psi$. By differentiating equation (6) with respect to time, we obtain

$$
\boldsymbol{v}_{O 2}=\left[\begin{array}{ccccc}
1 & 0 & 0 & d \cdot \mathrm{c} \theta \mathrm{c} \psi & -d \cdot \mathrm{s} \theta \mathrm{s} \psi \\
0 & 1 & 0 & 0 & -d \cdot \mathrm{c} \psi \\
0 & 0 & 1 & -d \cdot \mathrm{s} \theta \mathrm{c} \psi & -d \cdot \mathrm{c} \theta \mathrm{s} \psi
\end{array}\right] \boldsymbol{v}_{s}
$$

where

$$
\boldsymbol{v}_{s}=\left[\begin{array}{lllll}
\dot{x}_{D} & \dot{y}_{D} & \dot{z}_{D} & \dot{\theta} & \dot{\psi}
\end{array}\right]^{\mathrm{T}}
$$

The rotational angular velocity $\boldsymbol{\omega}$ of the MP can be expressed by the linear superposition of the Tait-Bryan angular velocity $\left[\begin{array}{lll}\dot{\phi} & \dot{\theta} & \dot{\psi}\end{array}\right]^{\mathrm{T}}$

$$
\boldsymbol{\omega}=\left[\begin{array}{ccc}
0 & -\mathrm{s} \phi & \mathrm{c} \phi \mathrm{c} \theta \\
0 & \mathrm{c} \phi & \mathrm{s} \phi \mathrm{c} \theta \\
1 & 0 & -\mathrm{s} \theta
\end{array}\right]\left[\begin{array}{c}
\dot{\phi} \\
\dot{\theta} \\
\dot{\psi}
\end{array}\right]
$$

As $\dot{\phi}=0$, the equation becomes

$$
\boldsymbol{\omega}=\left[\begin{array}{cc}
0 & \mathrm{c} \theta \\
1 & 0 \\
0 & -\mathrm{s} \theta
\end{array}\right]\left[\begin{array}{c}
\dot{\theta} \\
\dot{\psi}
\end{array}\right]=\left[\begin{array}{ccccc}
0 & 0 & 0 & 0 & \mathrm{c} \theta \\
0 & 0 & 0 & 1 & 0 \\
0 & 0 & 0 & 0 & -\mathrm{s} \theta
\end{array}\right] \boldsymbol{v}_{s}
$$

From equations (24) and (26), the following expression can be obtained:

$$
\left[\begin{array}{c}
\boldsymbol{v}_{O 2} \\
\boldsymbol{\omega}
\end{array}\right]=\boldsymbol{J}_{o} \boldsymbol{v}_{s}
$$

where

$$
\boldsymbol{J}_{o}=\left[\begin{array}{ccccc}
1 & 0 & 0 & d \cdot \mathrm{c} \theta \mathrm{c} \psi & -d \cdot \mathrm{s} \theta \mathrm{s} \psi \\
0 & 1 & 0 & 0 & -d \cdot \mathrm{c} \psi \\
0 & 0 & 1 & -d \cdot \mathrm{s} \theta \mathrm{c} \psi & -d \cdot \mathrm{c} \theta \mathrm{s} \psi \\
0 & 0 & 0 & 0 & \mathrm{c} \theta \\
0 & 0 & 0 & 1 & 0 \\
0 & 0 & 0 & 0 & -\mathrm{s} \theta
\end{array}\right]
$$

Substituting equation (28) into equation (22) yields

$$
\boldsymbol{v}_{l}=\boldsymbol{J}_{l} \boldsymbol{J}_{o} \boldsymbol{v}_{s}=\boldsymbol{J}_{4 \times 5} \boldsymbol{v}_{s}
$$

where $\boldsymbol{J}_{4 \times 5}$ is a $4 \times 5$ velocity Jacobian matrix. This reflects the mapping relationship between the driving velocity $v_{l i}(i=1,2,3,4)$ of the rods and variation velocity $\boldsymbol{v}_{s}$ of the generalized coordinates.

The $y$ axis component $y_{O 1}$ of the position vector $\boldsymbol{O}_{1}$ of the sliding platform center is regarded as the function of the generalized coordinates $x_{D}, y_{D}, z_{D}, \theta, \psi$. By differentiating $y_{O 1}$ of equation (7), the driving velocity $v_{O 1}$ along guide direction is obtained as

$$
v_{O 1}=\boldsymbol{J}_{O 1} \boldsymbol{v}_{s}
$$

where

$$
\boldsymbol{J}_{O 1}=\left[\begin{array}{lllll}
0 & 1 & 0 & 0 & -d \cdot c \psi
\end{array}\right]
$$

When $\dot{x}_{D}, \dot{y}_{D}, \dot{z}_{D}, \dot{\theta}, \dot{\psi}$ are known, the driving velocities of each driving rod and sliding platform can be obtained from equations (30) and (31).

The driving velocities obtained above are all relative to the coordinate system $\{O\}$. The driving velocities ${ }^{O_{1}} v_{l i}(i=1,2,3,4)$ of each rod relative to the sliding platform $\left\{O_{1}\right\}$ can be expressed as

$$
\begin{gathered}
{ }^{{ }^{1}} v_{l i}=v_{l i}-v_{O 1} \boldsymbol{n}_{O 1} \cdot \boldsymbol{n}_{i}=v_{l i}-\boldsymbol{J}_{O 1} \boldsymbol{v}_{s} \boldsymbol{n}_{O 1} \cdot \boldsymbol{n}_{i} \\
{ }^{{ }^{1}} \boldsymbol{v}_{l}=\boldsymbol{v}_{l}-\boldsymbol{J}_{O 1} \boldsymbol{v}_{s}\left[\begin{array}{c}
\boldsymbol{n}_{O 1} \cdot \boldsymbol{n}_{1} \\
\boldsymbol{n}_{O 1} \cdot \boldsymbol{n}_{2} \\
\boldsymbol{n}_{O 1} \cdot \boldsymbol{n}_{3} \\
\boldsymbol{n}_{O 1} \cdot \boldsymbol{n}_{4}
\end{array}\right]=\boldsymbol{J}_{4 \times 5}^{\prime} \boldsymbol{v}_{s} .
\end{gathered}
$$

where

$$
\boldsymbol{J}_{4 \times 5}^{\prime}=\left[\boldsymbol{J}_{4 \times 5}-\left[\begin{array}{c}
\boldsymbol{n}_{O 1} \cdot \boldsymbol{n}_{1} \\
\boldsymbol{n}_{O 1} \cdot \boldsymbol{n}_{2} \\
\boldsymbol{n}_{O 1} \cdot \boldsymbol{n}_{3} \\
\boldsymbol{n}_{O 1} \cdot \boldsymbol{n}_{4}
\end{array} \boldsymbol{J}_{O 1}\right]_{4 \times 5}\right.
$$

where $\boldsymbol{n}_{O 1}=\left[\begin{array}{lll}0 & 1 & 0\end{array}\right]^{T}$ is the unit vector in the $y$ axis direction.

\subsection{The Acceleration Analysis of the 5-DOF GHMT}

Suppose that $\boldsymbol{a}$ and $\boldsymbol{\varepsilon}$ are the corresponding linear acceleration vector and angular acceleration vector of the MP, respectively.

$$
\begin{aligned}
\boldsymbol{a} & =\left[\begin{array}{lll}
a_{x} & a_{y} & a_{z}
\end{array}\right]^{\mathrm{T}} \\
\boldsymbol{\varepsilon} & =\left[\begin{array}{lll}
\varepsilon_{x} & \varepsilon_{y} & \varepsilon_{z}
\end{array}\right]^{\mathrm{T}} \\
\boldsymbol{A} & =\left[\begin{array}{ll}
\boldsymbol{a} & \boldsymbol{\varepsilon}
\end{array}\right]^{\mathrm{T}}
\end{aligned}
$$

Suppose that there are 2 vectors $\boldsymbol{\eta}$ and $\boldsymbol{\varsigma}$ and a skewsymmetric matrix $\hat{\boldsymbol{\eta}}$ as follows:

$$
\boldsymbol{\eta}=\left[\begin{array}{l}
\eta_{x} \\
\eta_{y} \\
\eta_{z}
\end{array}\right], \boldsymbol{\varsigma}=\left[\begin{array}{c}
\varsigma_{x} \\
\varsigma_{y} \\
\varsigma_{z}
\end{array}\right], \hat{\boldsymbol{\eta}}=\left[\begin{array}{ccc}
0 & -\eta_{z} & \eta_{y} \\
\eta_{z} & 0 & -\eta_{x} \\
-\eta_{y} & \eta_{x} & 0
\end{array}\right]
$$


Suppose that $a_{l i}$ is the acceleration along the rods $\boldsymbol{n}_{i}(i=1,2,3,4)$ direction. By differentiating equation (21) with respect to time, the expression of $a_{l i}$ can be given as

$$
a_{l i}=\left[\begin{array}{ll}
\boldsymbol{n}_{i}^{\mathrm{T}} & \left(\boldsymbol{e}_{i} \times \boldsymbol{n}_{i}\right)^{\mathrm{T}}
\end{array}\right]\left[\begin{array}{l}
\boldsymbol{a} \\
\boldsymbol{\varepsilon}
\end{array}\right]+\left[\begin{array}{ll}
\boldsymbol{v}_{O 2}^{\mathrm{T}} & \boldsymbol{\omega}^{\mathrm{T}}
\end{array}\right] \boldsymbol{H}_{i}\left[\begin{array}{c}
\boldsymbol{v}_{O 2} \\
\boldsymbol{\omega}
\end{array}\right]
$$

where

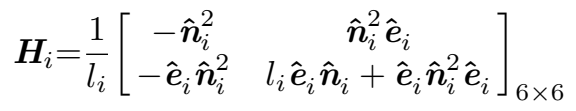

By differentiating equations (24) and (27) with respect to time and considering equations (28) and (29), we obtain the expression of acceleration $\boldsymbol{A}$ of the MP.

$$
\boldsymbol{A}=\boldsymbol{J}_{o} \boldsymbol{a}_{s}+\boldsymbol{v}_{s}^{\mathrm{T}} \boldsymbol{h} \boldsymbol{v}_{s}=\boldsymbol{J}_{o} \boldsymbol{a}_{s}+\left[\begin{array}{c}
\boldsymbol{v}_{s}^{\mathrm{T}} \boldsymbol{h}_{1} \boldsymbol{v}_{s} \\
\boldsymbol{v}_{s}^{\mathrm{T}} \boldsymbol{h}_{2} \boldsymbol{v}_{s} \\
\vdots \\
\boldsymbol{v}_{s}^{\mathrm{T}} \boldsymbol{h}_{6} \boldsymbol{v}_{s}
\end{array}\right]
$$

where

$$
\begin{aligned}
\boldsymbol{a}_{s} & =\left[\begin{array}{lllll}
\ddot{x}_{D} & \ddot{y}_{D} & \ddot{z}_{D} & \ddot{\theta} & \ddot{\psi}
\end{array}\right]^{\mathrm{T}} \\
\boldsymbol{h} & =\left[\begin{array}{llll}
\boldsymbol{h}_{1} & \boldsymbol{h}_{2} & \cdots & \boldsymbol{h}_{6}
\end{array}\right]^{\mathrm{T}}
\end{aligned}
$$

where $h_{i}$ is the $i$ th $5 \times 5$ Hessian matrix:

$$
\boldsymbol{h}_{1}=\left[\begin{array}{ccccc}
0 & 0 & 0 & 0 & 0 \\
0 & 0 & 0 & 0 & 0 \\
0 & 0 & 0 & 0 & 0 \\
0 & 0 & 0 & -d \cdot \mathrm{s} \theta \mathrm{c} \psi & -d \cdot \mathrm{c} \theta \mathrm{s} \psi \\
0 & 0 & 0 & -d \cdot \mathrm{c} \theta \mathrm{s} \psi & -d \cdot \mathrm{s} \theta \mathrm{c} \psi
\end{array}\right]
$$

$$
\begin{aligned}
& \boldsymbol{h}_{2}=\left[\begin{array}{ccccc}
0 & 0 & 0 & 0 & 0 \\
0 & 0 & 0 & 0 & 0 \\
0 & 0 & 0 & 0 & 0 \\
0 & 0 & 0 & 0 & 0 \\
0 & 0 & 0 & 0 & d \cdot \mathrm{s} \psi
\end{array}\right] \\
& \boldsymbol{h}_{3}=\left[\begin{array}{ccccc}
0 & 0 & 0 & 0 & 0 \\
0 & 0 & 0 & 0 & 0 \\
0 & 0 & 0 & 0 & 0 \\
0 & 0 & 0 & -d \cdot \mathrm{c} \theta \mathrm{c} \psi & d \cdot \mathrm{s} \theta \mathrm{s} \psi \\
0 & 0 & 0 & d \cdot \mathrm{s} \theta \mathrm{s} \psi & -d \cdot \mathrm{c} \theta \mathrm{c} \psi
\end{array}\right] \\
& \boldsymbol{h}_{4}=\left[\begin{array}{ccccc}
0 & 0 & 0 & 0 & 0 \\
0 & 0 & 0 & 0 & 0 \\
0 & 0 & 0 & 0 & 0 \\
0 & 0 & 0 & 0 & -\mathrm{s} \theta \\
0 & 0 & 0 & 0 & 0
\end{array}\right] \\
& \boldsymbol{h}_{5}=\left[\begin{array}{lllll}
0 & 0 & 0 & 0 & 0 \\
0 & 0 & 0 & 0 & 0 \\
0 & 0 & 0 & 0 & 0 \\
0 & 0 & 0 & 0 & 0 \\
0 & 0 & 0 & 0 & 0
\end{array}\right] \\
& \boldsymbol{h}_{6}=\left[\begin{array}{ccccc}
0 & 0 & 0 & 0 & 0 \\
0 & 0 & 0 & 0 & 0 \\
0 & 0 & 0 & 0 & 0 \\
0 & 0 & 0 & 0 & -\mathrm{c} \theta \\
0 & 0 & 0 & 0 & 0
\end{array}\right]
\end{aligned}
$$

By differentiating equation (31) with respect to time, the acceleration of the sliding platform along the guide direction can be expressed as

$$
a_{O 1}=\boldsymbol{J}_{O 1} \boldsymbol{a}_{s}+\boldsymbol{v}_{s}^{\mathrm{T}} \boldsymbol{h}_{O 1} \boldsymbol{v}_{s}
$$

where $\boldsymbol{h}_{O 1}$ is a $5 \times 5$ Hessian matrix.

$$
\boldsymbol{h}_{O 1}=\left[\begin{array}{ccccc}
0 & 0 & 0 & 0 & 0 \\
0 & 0 & 0 & 0 & 0 \\
0 & 0 & 0 & 0 & 0 \\
0 & 0 & 0 & 0 & 0 \\
0 & 0 & 0 & 0 & d \cdot \mathrm{s} \psi
\end{array}\right]
$$

When $\dot{x}_{D}, \dot{y}_{D}, \dot{z}_{D}, \dot{\theta}, \dot{\psi}$ and $\ddot{x}_{D}, \ddot{y}_{D}, \ddot{z}_{D}, \ddot{\theta}, \ddot{\psi}$ are known, the acceleration of each driving rod can be obtained from equations (37) and (42).

\section{The Dynamic Model of the 5-DOF GHMT}

The velocity of the end of each limb on the MP can be expressed as

$$
\boldsymbol{v}_{i}=\boldsymbol{\omega}_{l i} \times l_{i} \boldsymbol{n}_{i}+{ }^{O_{1}} v_{l i} \boldsymbol{n}_{i}+v_{O 1} \boldsymbol{n}_{O 1}
$$


where $\boldsymbol{\omega}_{l i}$ is the angular velocity of limb $i$, and $\boldsymbol{n}_{O 1}$ is the unit direction vector of the driving velocity along the guide direction.

Cross multiplying $\boldsymbol{n}_{i}$ at both sides of equation (44) gives

$$
\begin{aligned}
& \boldsymbol{n}_{i} \times \boldsymbol{v}_{i} \\
= & \boldsymbol{n}_{i} \times\left(\boldsymbol{\omega}_{l i} \times l_{i} \boldsymbol{n}_{i}\right)+\boldsymbol{n}_{i} \times{ }^{O_{1}} v_{l i} \boldsymbol{n}_{i}+\boldsymbol{n}_{i} \times v_{O 1} \boldsymbol{n}_{O 1} \\
= & l_{i} \boldsymbol{\omega}_{l i}-l_{i} \boldsymbol{n}_{i}\left(\boldsymbol{n}_{i} \cdot \boldsymbol{\omega}_{l i}\right)+\boldsymbol{n}_{i} \times v_{O 1} \boldsymbol{n}_{O 1}
\end{aligned}
$$

For the RPU limbs, i.e. limb $i(i=1,3)$ has no rotation around the rod, thus $\boldsymbol{\omega}_{l i} \cdot \boldsymbol{n}_{i}=0$.

$$
\begin{aligned}
\boldsymbol{\omega}_{l i} & =\frac{1}{l_{i}}\left(\boldsymbol{n}_{i} \times \boldsymbol{v}_{i}-\boldsymbol{n}_{i} \times v_{O 1} \boldsymbol{n}_{O 1}\right) \\
& =\frac{1}{l_{i}}\left(\boldsymbol{n}_{i} \times \boldsymbol{v}_{i}-v_{O 1} \hat{\boldsymbol{n}}_{i} \boldsymbol{n}_{O 1}\right)
\end{aligned}
$$

By substituting equations (20), (28) and (31) into equation (46), $\boldsymbol{\omega}_{l i}(i=1,3)$ can be derived as

$$
\begin{aligned}
& \boldsymbol{\omega}_{l i}=\frac{1}{l_{i}}\left\{\left[\boldsymbol{n}_{i} \times\left(\boldsymbol{v}_{O 2}+\boldsymbol{\omega} \times \boldsymbol{e}_{i}\right)\right]-\hat{\boldsymbol{n}}_{i} \boldsymbol{n}_{O 1} \boldsymbol{J}_{O 1} \boldsymbol{v}_{s}\right\} \\
& =\frac{1}{l_{i}}\left\{\left[\begin{array}{ll}
\hat{\boldsymbol{n}}_{i} & -\hat{\boldsymbol{n}}_{i} \hat{\boldsymbol{e}}_{i}
\end{array}\right] \boldsymbol{J}_{O}-\hat{\boldsymbol{n}}_{i} \boldsymbol{n}_{O 1} \boldsymbol{J}_{O 1}\right\} \boldsymbol{v}_{s} \\
& =\boldsymbol{J}_{\boldsymbol{\omega} i} \boldsymbol{v}_{s}
\end{aligned}
$$

where $i=1,3$, and

$$
\boldsymbol{J}_{\boldsymbol{\omega} i}=\frac{1}{l_{i}}\left\{\left[\begin{array}{ll}
\hat{\boldsymbol{n}}_{i} & -\hat{\boldsymbol{n}}_{i} \hat{\boldsymbol{e}}_{i}
\end{array}\right] \boldsymbol{J}_{o}-\hat{\boldsymbol{n}}_{i} \boldsymbol{n}_{O 1} \boldsymbol{J}_{O 1}\right\}
$$

By differentiating equation (46), the angular acceleration $\varepsilon_{l i}$ of limb $i(i=1,3)$ is

$$
\begin{aligned}
\varepsilon_{l i}= & \frac{\left[\dot{\boldsymbol{n}}_{i} \times \boldsymbol{v}_{i}+\boldsymbol{n}_{i} \times \dot{\boldsymbol{v}}_{i}-\left(a_{O 1} \boldsymbol{n}_{i}+v_{O 1} \dot{\boldsymbol{n}}_{i}\right) \times \boldsymbol{n}_{O 1}\right]}{l_{i}} \\
& -\frac{\boldsymbol{\omega}_{l i}{ }^{O_{1}} v_{l i}}{l_{i}}
\end{aligned}
$$

The two revolute pairs $\mathrm{R}$ of the Hooke hinges $\mathrm{U}$ on the UPS limbs, i.e. limb $i(i=2,4)$ are denoted as $R_{i j}$ $(j=1,2)$. The angular velocity of limb $i(i=2,4)$ can be expressed as:

$$
\boldsymbol{\omega}_{l i}=\dot{\theta}_{i 1} \boldsymbol{R}_{i 1}+\dot{\theta}_{i 2} \boldsymbol{R}_{i 2}, \quad \boldsymbol{R}_{i 2}=\frac{\boldsymbol{R}_{i 1} \times \boldsymbol{n}_{i}}{\left|\boldsymbol{R}_{i 1} \times \boldsymbol{n}_{i}\right|}
$$

where $\dot{\theta}_{i 1}, \dot{\theta}_{i 2}$ are magnitude of the angular velocities of the $\boldsymbol{R}_{i 1}, \boldsymbol{R}_{i 2}(i=2,4)$, respectively; $\boldsymbol{R}_{i 1}, \boldsymbol{R}_{i 1}$ are the unit direction vector of the $R_{i 1}, R_{i 2}$, respectively, where $\boldsymbol{R}_{i 1}=\left[\begin{array}{lll}1 & 0 & 0\end{array}\right]^{\mathrm{T}}$.

Cross multiplying $l_{i} \boldsymbol{n}_{i}(\mathrm{i}=2,4)$ at both sides of equation (50) gives

$$
\begin{aligned}
& \dot{\theta}_{i 1} \boldsymbol{R}_{i 1} \times l_{i} \boldsymbol{n}_{i}+\dot{\theta}_{i 2} \boldsymbol{R}_{i 2} \times l_{i} \boldsymbol{n}_{i} \\
& =\boldsymbol{\omega}_{l i} \times l_{i} \boldsymbol{n}_{i}=\boldsymbol{v}_{i}-{ }^{O_{1}} v_{l i} \boldsymbol{n}_{i}-v_{O 1} \boldsymbol{n}_{O 1} \\
& =-\left\{\left[\begin{array}{ll}
\hat{\boldsymbol{n}}_{i}^{2} & \left.-\hat{\boldsymbol{n}}_{i}^{2} \hat{\boldsymbol{e}}_{i}\right]
\end{array}\right] \boldsymbol{J}_{o}-\hat{\boldsymbol{n}}_{i}^{2} \boldsymbol{n}_{O 1} \boldsymbol{J}_{O 1}\right\} \boldsymbol{v}_{s}
\end{aligned}
$$

Dot multiplying $\boldsymbol{R}_{i 2}$ at both sides of equation (51), we obtain

$$
\begin{aligned}
& \dot{\theta}_{i 1}\left(\boldsymbol{R}_{i 1} \times l_{i} \boldsymbol{n}_{i}\right) \cdot \boldsymbol{R}_{i 2} \\
= & -\boldsymbol{R}_{i 2}^{\mathrm{T}}\left\{\left[\hat{\boldsymbol{n}}_{i}^{2}-\hat{\boldsymbol{n}}_{i}^{2} \hat{\boldsymbol{e}}_{i}\right] \boldsymbol{J}_{o}-\hat{\boldsymbol{n}}_{i}^{2} \boldsymbol{n}_{O 1} \boldsymbol{J}_{O 1}\right\} \boldsymbol{v}_{s}
\end{aligned}
$$

Dot multiplying $\boldsymbol{R}_{i 1}$ at both sides of equation (51), we obtain

$$
\begin{aligned}
& \dot{\theta}_{i 2}\left(\boldsymbol{R}_{i 2} \times l_{i} \boldsymbol{n}_{i}\right) \cdot \boldsymbol{R}_{i 1} \\
= & -\boldsymbol{R}_{i 1}^{\mathrm{T}}\left\{\left[\hat{\boldsymbol{n}}_{i}^{2}-\hat{\boldsymbol{n}}_{i}^{2} \hat{\boldsymbol{e}}_{i}\right] \boldsymbol{J}_{O}-\hat{\boldsymbol{n}}_{i}^{2} \boldsymbol{n}_{O 1} \boldsymbol{J}_{O 1}\right\} \boldsymbol{v}_{s}
\end{aligned}
$$

Equations (52), (53) lead to

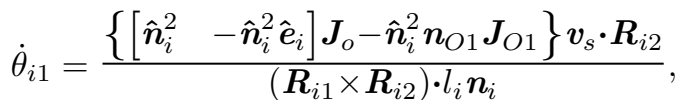

$$
\begin{aligned}
& \dot{\theta}_{i 2}=-\frac{\left\{\left[\hat{\boldsymbol{n}}_{i}^{2} \quad-\hat{\boldsymbol{n}}_{i}^{2} \hat{\boldsymbol{e}}_{i}\right] \boldsymbol{J}_{o}-\hat{\boldsymbol{n}}_{i}^{2} \boldsymbol{n}_{O 1} \boldsymbol{J}_{O 1}\right\} \boldsymbol{v}_{s} \cdot \boldsymbol{R}_{i 1}}{\left(\boldsymbol{R}_{i 1} \times \boldsymbol{R}_{i 2}\right) \cdot l_{i} \boldsymbol{n}_{i}}
\end{aligned}
$$

Combining equations (50), (54), (28), (31) and (33), we obtain

$$
\begin{aligned}
\boldsymbol{\omega}_{l i} & =\dot{\theta}_{i 1} \boldsymbol{R}_{i 1}+\dot{\theta}_{i 2} \boldsymbol{R}_{i 2} \\
& =\frac{\left(\boldsymbol{R}_{i 1} \times \boldsymbol{R}_{i 2}\right) \times\left(\boldsymbol{v}_{i}-{ }^{O_{1}} v_{l i} \boldsymbol{n}_{i}-v_{O 1} \boldsymbol{n}_{O 1}\right)}{l_{i} \boldsymbol{n}_{i} \cdot\left(\boldsymbol{R}_{i 1} \times \boldsymbol{R}_{i 2}\right)}
\end{aligned}
$$

where $i=2,4$.

$$
\begin{aligned}
\boldsymbol{J}_{\omega i}= & \frac{\left(\boldsymbol{R}_{i 1} \boldsymbol{R}_{i 2}^{\mathrm{T}}-\boldsymbol{R}_{i 2} \boldsymbol{R}_{i 1}^{\mathrm{T}}\right)\left\{\left[\hat{\boldsymbol{n}}_{i}^{2}-\hat{\boldsymbol{n}}_{\boldsymbol{i}}^{2} \hat{e}_{i}\right] J_{o}-\hat{\boldsymbol{n}}_{i}^{2} \boldsymbol{n}_{O 1} \boldsymbol{J}_{O 1}\right\}}{\left(\boldsymbol{R}_{i 1} \times \boldsymbol{R}_{i 2}\right) \cdot l_{i} \boldsymbol{n}_{i}} \\
& \left(\boldsymbol{R}_{i 1} \times \boldsymbol{R}_{i 2}\right) \times\left(\boldsymbol{v}_{i}-{ }^{O_{1}} v_{l i} \boldsymbol{n}_{i}-v_{O 1} \boldsymbol{n}_{O 1}\right) \\
= & \left(\boldsymbol{R}_{i 1} \boldsymbol{R}_{i 2}^{\mathrm{T}}-\boldsymbol{R}_{i 2} \boldsymbol{R}_{i 1}^{\mathrm{T}}\right) \\
& \left\{\left[\hat{\boldsymbol{n}}_{i}^{2}-\hat{\boldsymbol{n}}_{i}^{2} \hat{\boldsymbol{e}}_{i}\right] \boldsymbol{J}_{O}-\hat{\boldsymbol{n}}_{i}^{2} \boldsymbol{n}_{O 1} \boldsymbol{J}_{O 1}\right\} \boldsymbol{v}_{s}
\end{aligned}
$$

By differentiating equation (55), the angular acceleration $\varepsilon_{l i}$ of limb $i(i=2,4)$ is

$$
\begin{aligned}
& \boldsymbol{\varepsilon}_{l i}=\left\{\begin{array}{c}
-\dot{\theta}_{i 1} \boldsymbol{R}_{i 2} \times\left(\boldsymbol{v}_{i}-{ }^{O_{1}} v_{l i} \boldsymbol{n}_{i}-v_{O 1} \boldsymbol{n}_{O 1}\right) \\
+\left(\boldsymbol{R}_{i 1} \times \boldsymbol{R}_{i 2}\right) \\
\times\left(\begin{array}{l}
\boldsymbol{a}_{i}-{ }^{O_{1}} a_{l i} \boldsymbol{n}_{i}-{ }^{O_{1}} v_{l i} \boldsymbol{\omega}_{l i} \times \boldsymbol{n}_{i} \\
-a_{O 1} \boldsymbol{n}_{O 1}
\end{array}\right)
\end{array}\right\} \\
& \cdot\left(l_{i} \boldsymbol{n}_{i} \cdot\left(\boldsymbol{R}_{i 1} \times \boldsymbol{R}_{i 2}\right)\right)^{-1} \\
& -\boldsymbol{\omega}_{l i} \cdot\left\{\begin{array}{l}
\left(v_{l i} \boldsymbol{n}_{i}+l_{i} \boldsymbol{\omega}_{l i} \times \boldsymbol{n}_{i}\right) \cdot\left(\boldsymbol{R}_{i 1} \times \boldsymbol{R}_{i 2}\right) \\
-\dot{\theta}_{i 1} l_{i} \boldsymbol{n}_{i} \cdot \boldsymbol{R}_{i 2}
\end{array}\right\} \\
& \cdot\left(l_{i} \boldsymbol{n}_{i} \cdot\left(\boldsymbol{R}_{i 1} \times \boldsymbol{R}_{i 2}\right)\right)^{-1}
\end{aligned}
$$

The parameters of two components of the mechanism, the oscillating rod, and the telescopic rod, as shown in Figure 6, are listed in Table 2. 
Table 2: The kinematic parameters of the oscillating rod and the telescopic rod $(i=1,2,3,4)$

\begin{tabular}{|c|c|}
\hline Parameters & Description \\
\hline$l_{f i}$ & $\begin{array}{l}\text { distance from the oscillating rod } \\
\text { centroid to the end of the limb }\end{array}$ \\
\hline$l_{m i}$ & $\begin{array}{l}\text { distance from the telescopic rod } \\
\text { centroid to the end of the limb }\end{array}$ \\
\hline $\boldsymbol{v}_{f i}, \boldsymbol{a}_{f i}$ & $\begin{array}{l}\text { velocity and acceleration of the os- } \\
\text { cillating rod centroid, respectively }\end{array}$ \\
\hline $\boldsymbol{v}_{m i}, \boldsymbol{a}_{m i}$ & $\begin{array}{l}\text { velocity and acceleration of the } \\
\text { telescopic rod centroid, respec- } \\
\text { tively }\end{array}$ \\
\hline
\end{tabular}

Both the oscillating rod and the telescopic rod have the same angular velocity and angular acceleration.

The velocity of the oscillating rod centroid of each limb is

$$
\boldsymbol{v}_{f i}=\boldsymbol{\omega}_{l i} \times \boldsymbol{n}_{i} l_{f i}+v_{O 1} \boldsymbol{n}_{O 1}=\left(\boldsymbol{n}_{O 1} \boldsymbol{J}_{O 1}-l_{f i} \hat{\boldsymbol{n}}_{i} \boldsymbol{J}_{\omega i}\right) \boldsymbol{v}_{s}
$$

The following equations can be obtained.

$$
\left[\begin{array}{c}
\boldsymbol{v}_{f i} \\
\boldsymbol{\omega}_{l i}
\end{array}\right]=\boldsymbol{J}_{f i} \boldsymbol{v}_{s}
$$

where

$$
\boldsymbol{J}_{f i}=\left[\begin{array}{c}
\boldsymbol{n}_{O 1} \boldsymbol{J}_{O 1}-l_{f i} \hat{\boldsymbol{n}}_{i} \boldsymbol{J}_{\omega i} \\
\boldsymbol{J}_{\omega i}
\end{array}\right]
$$

The linear acceleration of the oscillating rod centroid of each limb is

$$
\boldsymbol{a}_{f i}=\varepsilon_{l i} \times \boldsymbol{n}_{i} l_{f i}+\boldsymbol{\omega}_{l i} \times\left(\boldsymbol{\omega}_{l i} \times \boldsymbol{n}_{i}\right) l_{f i}+\boldsymbol{n}_{O 1} a_{O 1}
$$

The velocity of the telescopic rod centroid of each limb is

$$
\begin{aligned}
\boldsymbol{v}_{m i}= & \boldsymbol{\omega}_{l i} \times \boldsymbol{n}_{i}\left(l_{i}-l_{m i}\right)+{ }^{O_{1}} v_{l i} \boldsymbol{n}_{i}+v_{O 1} \boldsymbol{n}_{O 1} \\
= & \left\{\begin{array}{l}
-\left(l_{i}-l_{m i}\right) \hat{\boldsymbol{n}}_{i} \boldsymbol{J}_{\omega i} \\
+\left[\boldsymbol{n}_{i} \boldsymbol{n}_{i}^{\mathrm{T}}-\boldsymbol{n}_{i} \boldsymbol{n}_{i}^{\mathrm{T}} \hat{\boldsymbol{e}}_{i}\right] \boldsymbol{J}_{O} \\
-\hat{\boldsymbol{n}}_{i}^{2} \boldsymbol{n}_{O 1} \boldsymbol{J}_{O 1}
\end{array}\right\} \boldsymbol{v}_{s}
\end{aligned}
$$

The following equations can then be obtained.

$$
\left[\begin{array}{c}
\boldsymbol{v}_{m i} \\
\boldsymbol{\omega}_{l i}
\end{array}\right]=\boldsymbol{J}_{m i} \boldsymbol{v}_{s}
$$

where

$$
\left.\boldsymbol{J}_{m i}=\left[\begin{array}{c}
-\left(l_{i}-l_{m i}\right) \hat{\boldsymbol{n}}_{i} \boldsymbol{J}_{\omega i} \\
+\left[\boldsymbol{n}_{i} \boldsymbol{n}_{i}^{\mathrm{T}}-\boldsymbol{n}_{i} \boldsymbol{n}_{i}^{\mathrm{T}} \hat{\boldsymbol{e}}_{i}\right] \boldsymbol{J}_{o} \\
-\hat{\boldsymbol{n}}_{i}^{2} \boldsymbol{n}_{O 1} \boldsymbol{J}_{O 1} \\
\boldsymbol{J}_{\omega i}
\end{array}\right\}\right]
$$

The linear acceleration $\boldsymbol{a}_{m i}(i=1,2,3,4)$ of telescopic rod centroid of each limb is

$$
\begin{aligned}
\boldsymbol{a}_{m i}= & \boldsymbol{\varepsilon}_{l i} \times \boldsymbol{n}_{i}\left(l_{i}-l_{m i}\right)+\boldsymbol{\omega}_{l i} \times\left(\boldsymbol{\omega}_{l i} \times \boldsymbol{n}_{i}\right)\left(l_{i}-l_{m i}\right) \\
& +{ }^{O_{1}} a_{l i} \boldsymbol{n}_{i}+2 \cdot\left(\boldsymbol{\omega}_{l i} \times \boldsymbol{n}_{i}\right){ }^{O_{1}} v_{l i}+a_{O 1} \boldsymbol{n}_{O 1}
\end{aligned}
$$

The dynamic parameters of the oscillating rod and the telescopic rod are shown in Table 3.

Table 3: The dynamic parameters of the oscillating rod and the telescopic rod $(i=1,2,3,4)$

\begin{tabular}{cl}
\hline Parameters & Description \\
\hline $\boldsymbol{G}_{f i}$ & $\begin{array}{l}\text { The gravity vector of the os- } \\
\text { cillating rod }\end{array}$ \\
& The inertia force and inertia \\
& moment of the oscillating rod, \\
& respectively \\
$\boldsymbol{G}_{m i}$ & The gravity vector of the tele- \\
& scopic rod \\
$\boldsymbol{f}_{m i}, \boldsymbol{n}_{m i}$ & The inertia force and inertia \\
& moment of the telescopic rod, \\
& respectively \\
$\boldsymbol{G}_{O 1}, \boldsymbol{G}_{O}$ & The gravity of the sliding plat- \\
& form and the MP, respectively \\
$\boldsymbol{f}_{O}, \boldsymbol{n}_{O}$ & The inertia force and inertia \\
& moment of MP, respectively \\
$\boldsymbol{f}_{O 1}$ & The inertia force of the sliding \\
& platform \\
$\boldsymbol{F}_{O}, \boldsymbol{T}_{O}$ & The external force and the ex- \\
& ternal moment applied to the \\
& MP, respectively \\
& The masses of the oscillating \\
$m_{f i}, m_{m i}$ & rod and the telescopic rod, re- \\
& spectively. \\
& The masses of the sliding plat- \\
& form and the MP, respectively \\
& The mass of the motorized \\
$m_{O 1}, m_{O 2}$ & spindle \\
$m_{\mathrm{d}}$ &
\end{tabular}

The relationships of the dynamic parameters in Table 3 are as follows:

$$
\boldsymbol{G}_{f i}=m_{f i} \boldsymbol{g}, \quad \boldsymbol{f}_{f i}=-m_{f i} \boldsymbol{a}_{f i}
$$

$$
\begin{aligned}
& \boldsymbol{G}_{m i}=m_{m i} \boldsymbol{g}, \quad \boldsymbol{f}_{m i}=-m_{m i} \boldsymbol{a}_{m i} \\
& \boldsymbol{G}_{O 1}=m_{O 1} \boldsymbol{g}, \quad \boldsymbol{G}_{O}=\left(m_{O 2}+m_{\mathrm{d}}\right) \boldsymbol{g}
\end{aligned}
$$

$\boldsymbol{f}_{O 1}=-m_{O 1} \boldsymbol{a}_{O 1}=-m_{O 1} a_{O 1} \mathbf{n}_{O 1}$

$\boldsymbol{f}_{O}=-\left(m_{O 2}+m_{\mathrm{d}}\right) \boldsymbol{a}, \boldsymbol{m}_{i}=\frac{\boldsymbol{r}_{i}}{\left|\boldsymbol{r}_{i}\right|}$

$$
\boldsymbol{n}_{f i}=-{ }_{D i}^{O} \boldsymbol{R}^{i} \boldsymbol{I}_{f i}{ }_{D i}^{O} \boldsymbol{R}^{\mathrm{T}} \boldsymbol{\varepsilon}_{l i}-\boldsymbol{\omega}_{l i} \times\left({ }_{D i}^{O} \boldsymbol{R}^{i} \boldsymbol{I}_{f i}{ }_{D i}^{O} \boldsymbol{R}^{\mathrm{T}} \boldsymbol{\omega}_{l i}\right)
$$




$$
\begin{aligned}
& \boldsymbol{n}_{O}=-\boldsymbol{R}\left({ }^{O 2} \boldsymbol{I}_{O 2}+{ }^{O 2} \boldsymbol{I}_{\mathrm{d}}\right) \boldsymbol{R}^{\mathrm{T}} \boldsymbol{\varepsilon} \\
& -\boldsymbol{\omega} \times\left[\boldsymbol{R}\left({ }^{O 2} \boldsymbol{I}_{O 2}+{ }^{O 2} \boldsymbol{I}_{\mathrm{d}}\right) \boldsymbol{R}^{\mathrm{T}} \boldsymbol{\omega}\right] \\
& \boldsymbol{n}_{m i}=-{ }_{D i}^{O} \boldsymbol{R}^{i} \boldsymbol{I}_{m i} \stackrel{O}{O} \boldsymbol{R}^{\mathrm{T}} \boldsymbol{\varepsilon}_{l i}-\boldsymbol{\omega}_{l i} \times\left({ }_{D i}^{O} \boldsymbol{R}^{i} \boldsymbol{I}_{m i} \underset{D i}{O} \boldsymbol{R}^{\mathrm{T}} \boldsymbol{\omega}_{l i}\right) \\
& { }_{D i}^{O} \boldsymbol{R}=\left[\begin{array}{lll}
\boldsymbol{n}_{i} \times \boldsymbol{m}_{i} & \boldsymbol{n}_{i} \times\left(\boldsymbol{n}_{i} \times \boldsymbol{m}_{i}\right) & \boldsymbol{n}_{i}
\end{array}\right]
\end{aligned}
$$

where ${ }^{O 2} \boldsymbol{I}_{\mathrm{O} 2}$ and ${ }^{\mathrm{O} 2} \boldsymbol{I}_{d}$ are the moments of inertia of the MP and motorized spindle relative to the moving coordinate system $\left\{\mathrm{O}_{2}\right\} ;{ }^{i} \boldsymbol{I}_{f i}$ and ${ }^{i} \boldsymbol{I}_{m i}$ are the moments of inertia of the oscillating rod and telescopic rod of the each limb relative to the centroid body-fixed coordinate system $\left\{D_{i}\right\}$. Moreover, ${ }_{D i}^{O} \boldsymbol{R}$ is the rotation matrix of the centroid body-fixed coordinate system relative to the coordinate system $\{O\}$.

The centroid body-fixed coordinate system $\left\{D_{i}\right\}$ is connected to the centroid of each limb, where $z_{D i}$ axis is parallel to $\boldsymbol{n}_{i}$, while $x_{D i}$ axis is parallel to $\boldsymbol{n}_{i} \times \boldsymbol{m}_{i}$.

Based on the principle of virtual work, the following equation was obtained:

$$
\begin{aligned}
& \boldsymbol{F}_{q}^{\mathrm{T} O_{1}} \boldsymbol{v}_{l}+F_{O 1} v_{O 1}+\left(\boldsymbol{f}_{O 1}+\boldsymbol{G}_{O 1}\right) \cdot v_{O 1} \boldsymbol{n}_{O 1} \\
& +\left[\begin{array}{cc}
\boldsymbol{F}_{O}^{\mathrm{T}}+\boldsymbol{f}_{O}^{\mathrm{T}}+\boldsymbol{G}_{O}^{\mathrm{T}} & \boldsymbol{T}_{O}^{\mathrm{T}}+\boldsymbol{n}_{O}^{\mathrm{T}}
\end{array}\right]\left[\begin{array}{c}
\boldsymbol{v}_{O 2} \\
\boldsymbol{\omega}
\end{array}\right] \\
& +\sum_{i=1}^{i=4}\left(\begin{array}{c}
{\left[\begin{array}{ll}
\boldsymbol{f}_{f i}^{\mathrm{T}}+\boldsymbol{G}_{f i}^{\mathrm{T}} & \boldsymbol{n}_{f i}^{\mathrm{T}}
\end{array}\right]\left[\begin{array}{c}
\boldsymbol{v}_{f i} \\
\boldsymbol{\omega}_{l i}
\end{array}\right]} \\
+\left[\begin{array}{ll}
\boldsymbol{f}_{m i}^{\mathrm{T}}+\boldsymbol{G}_{m i}^{\mathrm{T}} & \boldsymbol{n}_{m i}^{\mathrm{T}}
\end{array}\right]\left[\begin{array}{c}
\boldsymbol{v}_{m i} \\
\boldsymbol{\omega}_{l i}
\end{array}\right]
\end{array}\right)=0
\end{aligned}
$$

where $\boldsymbol{F}_{q}=\left[\begin{array}{llll}F_{q 1} & F_{q 2} & F_{q 3} & F_{q 4}\end{array}\right]^{\mathrm{T}}$ is the fourdimensional vector comprised of the driving force of each rod in the mechanism, and $F_{O 1}$ is the driving force along the guide direction.

By substituting equations (59) and (63) into equation (67) and combining equations (28) and (31), we obtain

$$
\begin{aligned}
& \boldsymbol{F}_{q}^{\mathrm{T} O_{1}} \boldsymbol{v}_{l}+F_{O 1} \boldsymbol{J}_{O 1} \boldsymbol{v}_{s}+\boldsymbol{J}_{O 1} \boldsymbol{v}_{s}\left(\left(\boldsymbol{f}_{O 1}+\boldsymbol{G}_{O 1}\right) \cdot \boldsymbol{n}_{O 1}\right) \\
+ & {\left[\begin{array}{ll}
\boldsymbol{F}_{O}^{\mathrm{T}}+\boldsymbol{f}_{O}^{\mathrm{T}}+\boldsymbol{G}_{O}^{\mathrm{T}} & \boldsymbol{T}_{O}^{\mathrm{T}}+\boldsymbol{n}_{O}^{\mathrm{T}}
\end{array}\right] \boldsymbol{J}_{o} \boldsymbol{v}_{s} } \\
+ & \sum_{i=1}^{i=4}\left(\begin{array}{cc}
{\left[\begin{array}{ll}
\boldsymbol{f}_{f i}^{\mathrm{T}}+\boldsymbol{G}_{f i}^{\mathrm{T}} & \boldsymbol{n}_{f i}^{\mathrm{T}}
\end{array}\right] \boldsymbol{J}_{f i} \boldsymbol{v}_{s}} \\
+\left[\begin{array}{ll}
\boldsymbol{f}_{m i}^{\mathrm{T}}+\boldsymbol{G}_{m i}^{\mathrm{T}} & \boldsymbol{n}_{m i}^{\mathrm{T}}
\end{array}\right] \boldsymbol{J}_{m i} \boldsymbol{v}_{s}
\end{array}\right)=0
\end{aligned}
$$

From equations (68) and (33), the following equation is obtained:

$$
\begin{aligned}
& {\left[\begin{array}{c}
\boldsymbol{F}_{q} \\
F_{O 1}
\end{array}\right]=} \\
& -\left(\left[\begin{array}{c}
\boldsymbol{J}_{4 \times 5}^{\prime} \\
\boldsymbol{J}_{O 1}
\end{array}\right]_{5 \times 5}^{-1}\right)^{\mathrm{T}}
\end{aligned}
$$

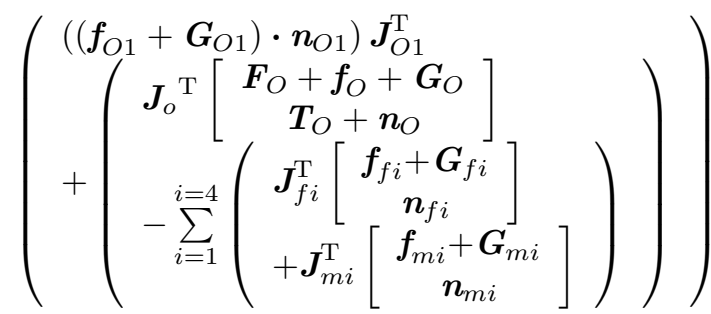

The dynamics of the mechanism can be solved from equation (69).

\section{Verification of the Dynamic Model}

\subsection{Dynamics Simulation with Matlab}

With the developed model, dynamics simulation was conducted with Matlab. The parameters of the machine tool are shown in Table 4 . With the parameters, the ranges of motion of the machine tool are determined, as listed in Table 5.

Table 4: The parameters of the machine tool $(i=1,2$, $3,4)$

\begin{tabular}{cc}
\hline Parameters & Value \\
\hline$a, b, c, d$ & $1.025 \mathrm{~m}, 0.925 \mathrm{~m}, 0.425 \mathrm{~m}$, \\
$l_{f i}, l_{m i}$ & $0.470 \mathrm{~m}$ \\
$l_{10}, l_{20}, l_{30}, l_{40}$ & $1.765 \mathrm{~m}, 1.790 \mathrm{~m}, 1.790 \mathrm{~m}$, \\
$l_{1 \mathrm{max}}, l_{1 \text { min }}$ & $1.790 \mathrm{~m}$ \\
$l_{2 \mathrm{max}}, l_{3 \max }, l_{4 \max }$ & $1.915 \mathrm{~m}, 1.915 \mathrm{~m}, 1.915 \mathrm{~m}$ \\
$l_{2 \mathrm{~min}}, l_{3 \min }, l_{4 \mathrm{~min}}$ & $1.665 \mathrm{~m}, 1.665 \mathrm{~m}, 1.665 \mathrm{~m}$ \\
$m_{O 1}, m_{O 2}$ & $1405.35 \mathrm{~kg}, 265.20 \mathrm{~kg}$ \\
$m_{d}, m_{m i}, m_{f i}$ & $77.91 \mathrm{~kg}, 60.95 \mathrm{~kg}, 86.53 \mathrm{~kg}$ \\
${ }^{O 2} \boldsymbol{I}_{O 2}$ & $\operatorname{diag}(13.7710 .3523 .67) \mathrm{kg} \cdot \mathrm{m}^{2}$ \\
${ }^{O 2} \boldsymbol{I}_{d}$ & $\operatorname{diag}(0.720 .720 .61) \mathrm{kg} \cdot \mathrm{m}{ }^{2}$ \\
${ }^{i} \boldsymbol{I}_{m i}$ & $\operatorname{diag}(15.4115 .410 .14) \mathrm{kg} \cdot \mathrm{m}^{2}$ \\
${ }^{i} \boldsymbol{I}_{f i}$ & $\operatorname{diag}(27.7827 .780 .17) \mathrm{kg} \cdot \mathrm{m}^{2}$ \\
$\boldsymbol{F}_{O}$ & {$[500500500]^{\mathrm{T}} \mathrm{N}$} \\
$\boldsymbol{T}_{O}$ & {$[500500500]^{\mathrm{T}} \mathrm{N} \cdot \mathrm{m}$} \\
\hline
\end{tabular}

Assuming the cutter moves from the initial position and orientation $\left(0,0,-2.154 \mathrm{~m}, 0^{\circ}, 0^{\circ}\right)$ with different 
accelerations (here, $0.1 \mathrm{~m} / \mathrm{s}^{2}, 0.1 \mathrm{~m} / \mathrm{s}^{2},-0.1 \mathrm{~m} / \mathrm{s}^{2}$, $4^{\circ} / \mathrm{s}^{2}$, and $4^{\circ} / \mathrm{s}^{2}$, respectively). By substituting the parameters from Table 4 into the kinematic model and dynamic model, we obtained the inverse solutions of the position, velocity, the acceleration, and the variation curve of the driving force with time using Matlab programming (Figure 7).

Table 5: The range of motion of the MP

\begin{tabular}{|c|c|}
\hline Description & Value \\
\hline $\begin{array}{l}\text { The range of } z \text { - } \\
\text { displacement }\end{array}$ & {$[-0.131 \mathrm{~m}, 0.133 \mathrm{~m}]$} \\
\hline $\begin{array}{l}\text { The maximum and mini- } \\
\text { mum displacements along } x \\
\text { direction at } z=-1.684 \mathrm{~m} \text {. }\end{array}$ & $-0.357 \mathrm{~m}, 0.787 \mathrm{~m}$ \\
\hline $\begin{array}{l}\text { The maximum and mini- } \\
\text { mum angles around } x \text { axis } \\
\text { at } z=-1.684 \mathrm{~m} \text {. }\end{array}$ & $-17.522^{\circ}, \quad 17.522^{\circ}$ \\
\hline $\begin{array}{l}\text { The maximum and mini- } \\
\text { mum angles around } y \text { axis } \\
\text { at } z=-1.684 \mathrm{~m} \text {. }\end{array}$ & $-18.194^{\circ}, \quad 17.522^{\circ}$ \\
\hline
\end{tabular}

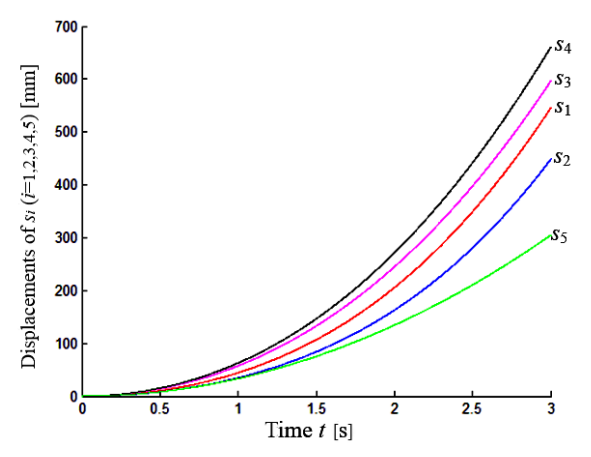

(a) Variation of the displacements

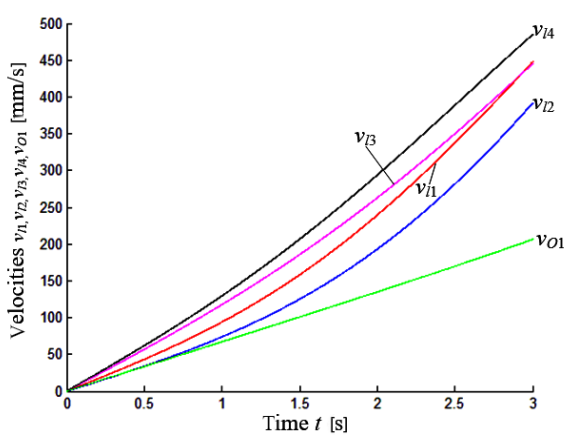

(b) Variation of the velocities

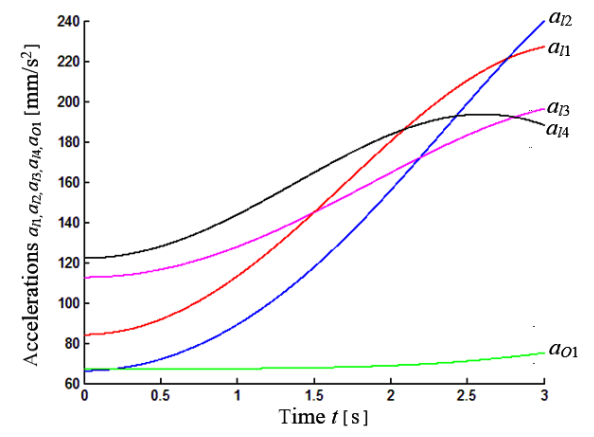

(c) Variation of the accelerations

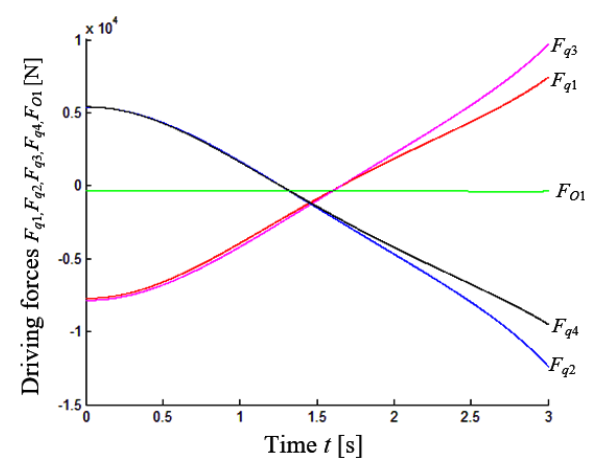

(d) Variation of the driving forces

Figure 7: The kinematics and dynamics results of the GHMT obtained from developed model

\subsection{Dynamic Simulation with ADAMS}

An ADAMS model was also developed with the defined material properties and the kinematic pairs. The same motion described in Section 5.1 is used to drive the mechanism. The inverse solutions of the position, velocity, the acceleration, and the variation curves of the driving forces were obtained in the post-processing module, as shown in Figure 8.

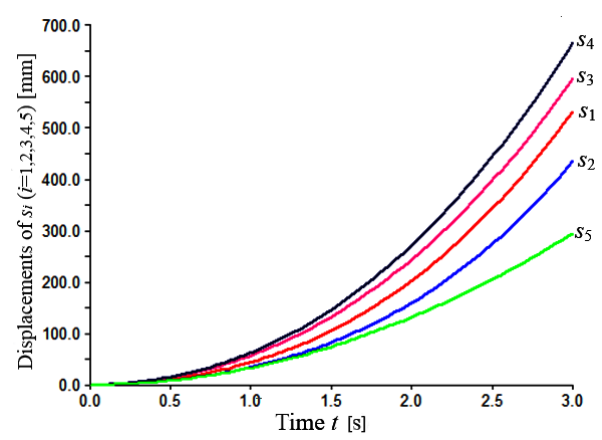

(a) Variation of the displacements 


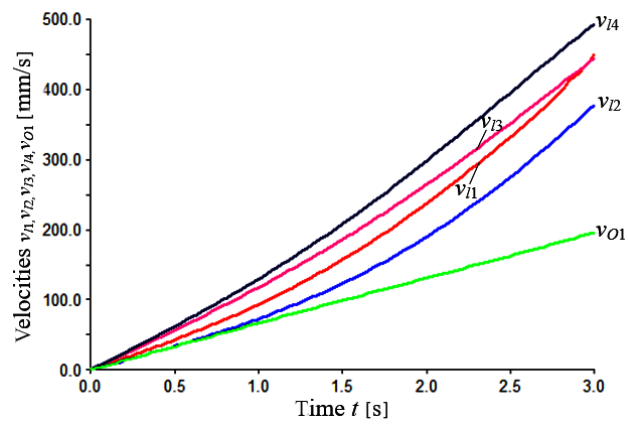

(b) Variation of the velocities

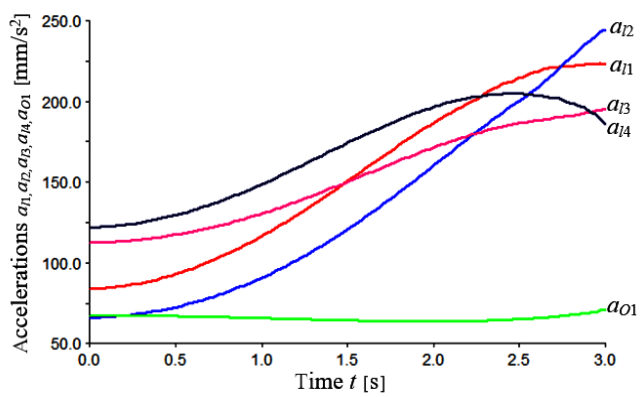

(c) Variation of the accelerations

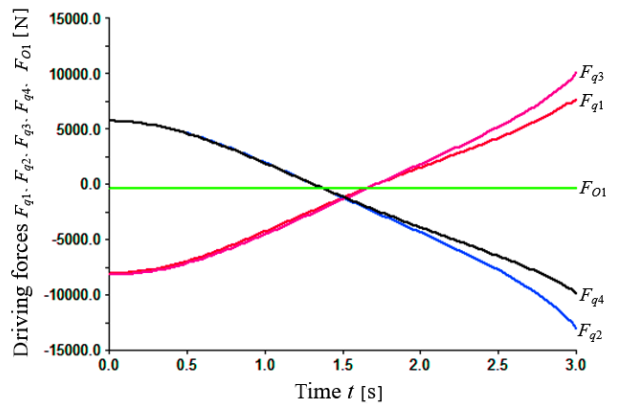

(d) Variation of the driving forces

Figure 8: The kinematics and dynamics results of the GHMT obtained from ADAMS

We found that the kinematic and dynamic results obtained from analytical model of the GHMT in Figure 7 and those results using the ADAMS simulation showing in Figure 8 agree generally well. There are very small difference among them, which was caused by errors, such as the establishment of the machine tool model, measurements, and the ADAMS fitting driving function.

Maximum error value occurs at driving force $\boldsymbol{F}_{O 1}$, which is equal to $4.6 \%$. The rest error values are in the range of $4 \%$. The results are consistent within the error range, which further shows the validity of the kinematic and dynamic models of the GHMT.

\section{A Case Study}

Figure 9 shows a drilling hole operation planned on a slender structural part, with a machining simulation model showing in Figure 10.

The machining process is described in Table 6 for the machining of two holes. By repeating Steps 2-7, the machining process for the rest of the holes is completed. We assumed that the process was uniform in every step, so the $\psi$ and $\theta$ angles of the MP were always 0 , and the resistance of the cutter along the positive $z$ axis direction in the drilling process was always $F_{O}=10000$ $\mathrm{N}$.

Let the parameters of the machine tool take the values in Table 4. Using the path identified in Table 6, the motion equations were programmed based on ADAMS. The variation curves of the displacements, velocities, accelerations, and driving forces of the hybrid machine tool were obtained, as shown in Figures 11 and 12.

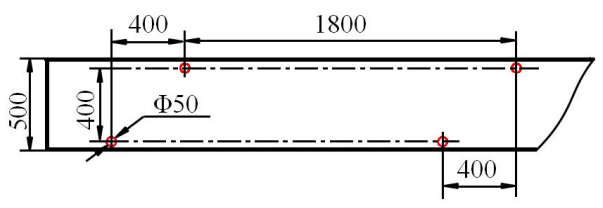

Figure 9: The slender structural part

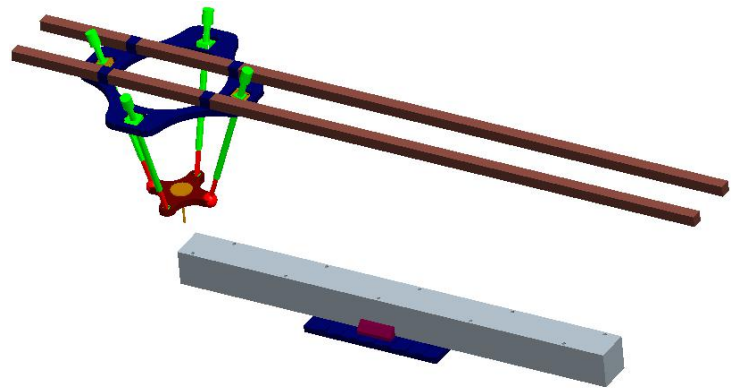

Figure 10: The machining simulation model

Figure 11 shows the variation laws of the displacement, velocity, and acceleration when the hybrid machine tool drills the first 4 holes. Analyzing Figure 9 reveals that for the whole process, the displacement $s_{2}$, velocity $v_{l 2}$, and acceleration $a_{l 2}$ were equal to the displacement $s_{4}$, velocity $v_{l 4}$, and acceleration $a_{l 4}$, respectively. This is consistent with the structural symmetry and motion symmetry of limbs 2 and 4 .

The displacement $s_{1}$, velocity $v_{l 1}$, and acceleration $a_{l 1}$ are different from the displacement $s_{3}$, velocity $v_{l 3}$, and acceleration $a_{l 3}$, respectively. This is consistent 
Table 6: The processing steps for drilling holes

\begin{tabular}{|c|c|c|c|c|}
\hline Step & $\begin{array}{l}\text { Displacements of the } \\
\text { cutter } / \mathrm{mm}\end{array}$ & $\begin{array}{l}\text { Moving direction } \\
\text { of the cutter }\end{array}$ & $\begin{array}{l}\text { Running } \\
\text { time/s }\end{array}$ & Executive action of the cutter \\
\hline 1 & $500 / 200 / 200$ & $-z /+x /+y$ & 3 & $\begin{array}{c}\text { Reaching the top of the first hole from } \\
\text { the initial position }\end{array}$ \\
\hline 2 & 300 & $-z$ & 5 & Drilling the first hole \\
\hline 3 & 300 & $+z$ & 3 & Retracting the cutter \\
\hline 4 & $400 / 400$ & $-x /+y$ & 4 & Reaching the top of the second hole \\
\hline 5 & 300 & $-z$ & 5 & Drilling the second hole \\
\hline 6 & 300 & $+z$ & 3 & Retracting the cutter \\
\hline 7 & $400 / 1000$ & $+x /+y$ & 5 & Reaching the top of the third hole \\
\hline
\end{tabular}

with the structural parameters $a \neq b$ in the sliding platform and the motion asymmetry of limbs 1 and 3 .

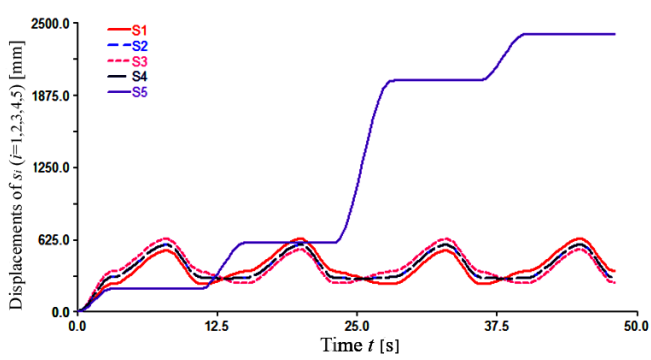

(a) The displacement

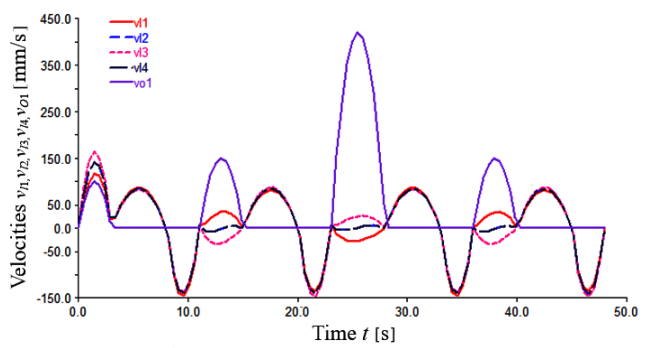

(b) The velocity

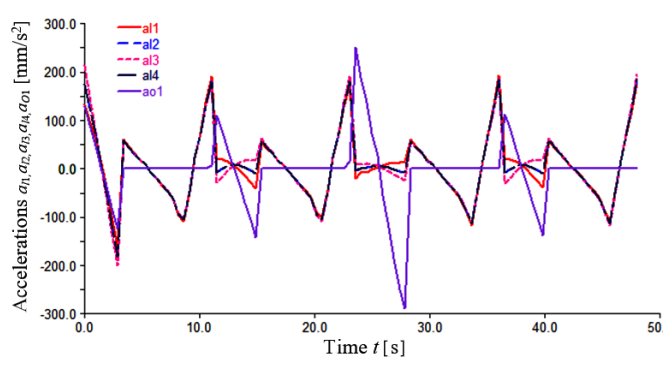

(c) The acceleration

Figure 11: The kinematics and dynamics results of the GHMT obtained from ADAMS

In the $3-8 \mathrm{~s}, 15-20 \mathrm{~s}, 28-33 \mathrm{~s}$, and $40-45 \mathrm{~s}$ drilling stages and in the 8-11 s, 20-23 s, 33-36 s, and 4548 s retracting stages, the variation tendencies of the displacement, velocity, and acceleration of the parallel mechanism are basically the same. We can clearly distinguish the 4 hole-drilling processes and cutter retraction processes. The direction of the guide did not change and its velocity and acceleration vanish. This coincides with the actual processing actions.

In the $0-3 \mathrm{~s}, 11-15 \mathrm{~s}, 23-28 \mathrm{~s}$, and $36-40 \mathrm{~s}$ movement stages along the guide direction, the differences between limbs 1 and 3 and between limbs 2 and 4 are obvious. There are velocity and acceleration variations along the guide direction. There is no violent fluctuation in the position, velocity, and acceleration throughout the whole process. This indicates that the dynamic performance of the machine tool is better than other machine tools that are currently available.

The variation of the driving forces of the machine tool is shown in Figure 12. The magnitudes of the driving forces $\boldsymbol{F}_{q 2}$ and $\boldsymbol{F}_{q 4}$ are equal throughout the machining process, which is in agreement with the situation where the structure and motion of limbs 2 and 4 are symmetrical. The magnitudes of the driving forces $\boldsymbol{F}_{q 1}$ and $\boldsymbol{F}_{q 3}$ are different, which is in agreement with the difference of the distance $a \neq b$ between the limbs 1 and 3 and the center in the sliding platform and movement difference.

The driving force $\boldsymbol{F}_{O 1}$ along the guide direction is small compared with the driving force $\boldsymbol{F}_{q i}$, thus the load is small along the guide direction. 


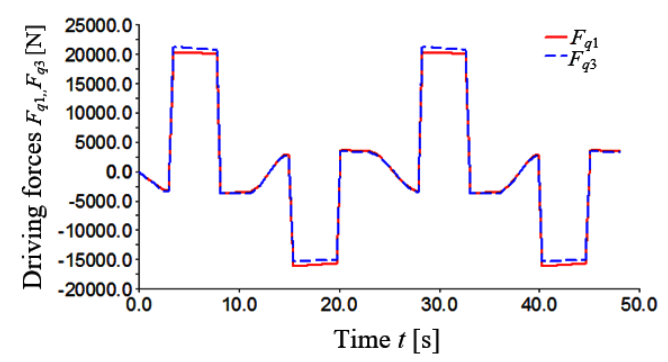

(a) The variations of the driving forces $\boldsymbol{F}_{q 1}$ and $\boldsymbol{F}_{q 3}$

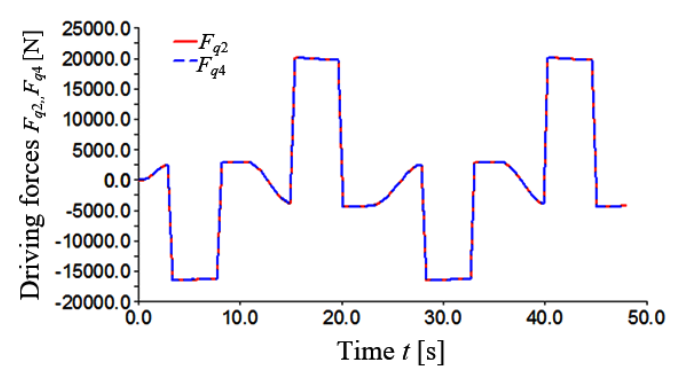

(b) The variations of the driving forces $\boldsymbol{F}_{q 2}$ and $\boldsymbol{F}_{q 4}$

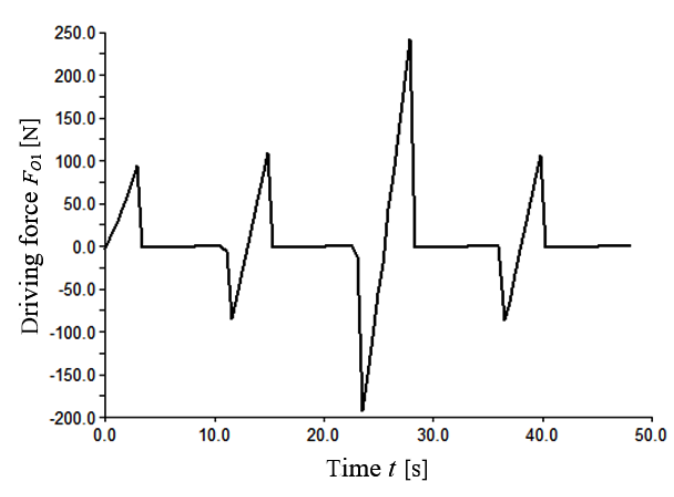

(c) The variation of the driving force $\boldsymbol{F}_{O 1}$

Figure 12: The variations of the driving forces

The driving forces $\boldsymbol{F}_{q i}(i=1,2,3,4)$ are the largest and relatively stable in the $3-8 \mathrm{~s}, 15-20 \mathrm{~s}, 28-33 \mathrm{~s}$, and 40-45s drilling stages. The driving forces $\boldsymbol{F}_{q i}(i=1,2,3$, 4) are smaller and relatively stable in the 8-11 s, 20-23 $\mathrm{s}, 33-36 \mathrm{~s}$, and $45-48 \mathrm{~s}$ cutter retraction stages, which is in agreement with practical engineering. The driving force $\boldsymbol{F}_{O 1}$ has obvious changes in the 0-3 s, 11-15 s, 23-28 s, and 36-40 s guide sliding stages. The driving force $\boldsymbol{F}_{O 1}$ drives the 2-RPU+2-UPS parallel structure to move.

\section{Discussion and Conclusions}

In this work, the dynamic modeling of a hybrid manipulator is studied, with due consideration on both kinematics and dynamics. The modeling work is conducted for a novel 3T2R GHMT of a 2-RPU+2-UPS configuration. The dynamic model of the machine tool was developed using the principle of virtual work based on the kinematic model. The model was validated by comparing the modeling results with MSC Adams simulation. A case study is included to show the application of the model in the determination of driving forces in machining.

The 5-DOF hybrid manipulator is proposed for machining of long structures. For machining applications, the dynamic performance is affected by a number of factors, for example, the workpiece material properties, the stiffness of the machine, among others. This paper focuses on the dynamics of the manipulator only. More comprehensive study on machining dynamics could be further considered upon the model developed in this work. In this light, the stiffness modeling of the manipulator is needed and can be obtained with a variety of available approaches (Wu et al. (2015a), Pashkevich et al. (2009)). These works are tasks of future study.

\section{Acknowledgements}

The authors gratefully acknowledge the financial support provided by the National Natural Science Foundation of China (Grant No. 51275486).

\section{References}

Assal, S. F. M. A novel planar parallel manipulator with high orientation capability for a hybrid machine tool: kinematics, dimensional synthesis and performance evaluation. Robotica, 2015. 35(5):1031-1053. doi:10.1017/S0263574715000958.

Cheng, G. and Shan, X. Dynamics analysis of a parallel hip joint simulator with four degree of freedoms (3R1T). Nonlinear Dynamics, 2012. 70(4):24752486. doi:10.1007/s11071-012-0635-4.

Gallardo-Alvarado, J., Aguilar-Njera, C. R., CasiqueRosas, L., Rico-Martnez, J. M., and Islam, M. N. Kinematics and dynamics of 2(3RPS) manipulators by means of screw theory and the principle of virtual work. Mechanism and Machine Theory, 2008. 43(10):1281-1294. doi:10.1016/j.mechmachtheory.2007.10.009.

Gallardo-Alvarado, J., Rodriguezcastro, R., Aguilarnajera, C. R., and Perezgonzalez, L. A novel six-degrees-of-freedom series-parallel manipulator. Journal of Mechanical Science and Technology, 2012. 26(6):1901-1909. doi:10.1007/s12206-012-0408-5. 
Gao, Z. and Zhang, D. Performance analysis, mapping, and multiobjective optimization of a hybrid robotic machine tool. Industrial Electronics IEEE Transactions on, 2015. 62(1):423-433. doi:10.1109/TIE.2014.2327008.

Horn, G. and Linge, S. Analytical generation of the dynamical equations for mechanical manipulators. Modeling Identification and Control, 1995. 16(3):155-167. doi:10.4173/mic.1995.3.4.

Hu, B., Lu, Y., Tan, Q., Yu, J., and Han, J. Analysis of stiffness and elastic deformation of a $2(\mathrm{SP}+\mathrm{SPR}+\mathrm{SPU})$ serial-parallel manipulator. Robotics and Computer-integrated Manufacturing, 2011. 27(2):418-425. doi:10.1016/j.rcim.2010.09.002.

Hu, B., Lu, Y., Yu, J., and Zhuang, S. Analyses of inverse kinematics, statics and workspace of a novel 3RPS-3SPR serialparallel manipulator. The Open Mechanical Engineering Journal, 2012. 6(Suppl.1):65-72. doi:10.2174/1874155X01206010065.

$\mathrm{Hu}$, B. and $\mathrm{Yu}$, J. Unified solving inverse dynamics of 6-DOF serialcparallel manipulators. Applied Mathematical Modelling, 2015. 39(16):4715-4732. doi:10.1016/j.apm.2015.04.020.

Huang, P., Wang, J., Wang, L., and Yao, R. Kinematical calibration of a hybrid machine tool with regularization method. International Journal of $M a-$ chine Tools and Manufacture, 2011. 51(3):210-220. doi:10.1016/j.ijmachtools.2010.11.009.

Huang, T., Wang, P., Zhao, X., and Chetwynd, D. G. Design of a 4-DOF hybrid pkm module for large structural component assembly. Cirp Annalsmanufacturing Technology, 2010. 59(1):159-162. doi:10.1016/j.cirp.2010.03.098.

Jalón, J. G. d. and Bayo, E. Kinematic and Dynamic Simulation of Multibody Systems: The Real Time Challenge. Springer-Verlag New York, Inc., Secaucus, NJ, USA, 1994.

Jiang, Y., Li, T., and Wang, L. Dynamic modeling and redundant force optimization of a 2-DOF parallel kinematic machine with kinematic redundancy. Robotics and Computer-integrated Manufacturing, 2015. 32:1-10. doi:10.1016/j.rcim.2014.08.001.

Khalil, W. and Ibrahim, O. General solution for the dynamic modeling of parallel robots. Journal of Intelligent and Robotic Systems, 2007. 49(1):19-37. doi:10.1007/s10846-007-9137-x.
Li, Y., Wang, J., Liu, X. J., and Wang, L. P. Dynamic performance comparison and counterweight optimization of two 3-DOF parallel manipulators for a new hybrid machine tool. Mechanism and Machine Theory, 2010. 45(11):1668-1680. doi:10.1016/j.mechmachtheory.2010.06.009.

Lian, B., Sun, T., Song, Y., Jin, Y., and Price, M. Stiffness analysis and experiment of a novel 5DOF parallel kinematic machine considering gravitational effects. International Journal of $M a$ chine Tools and Manufacture, 2015. 95:82-96. doi:10.1016/j.ijmachtools.2015.04.012.

Liang, C. and Ceccarelli, M. Design and simulation of a waist-trunk system for a humanoid robot. Mechanism and Machine Theory, 2012. 53:50-65. doi:10.1016/j.mechmachtheory.2012.02.009.

Liu, S. and $\mathrm{Yu}, \mathrm{Y}$. Dynamic design of a planar 3-DOF parallel manipulator. Chinese Journal of Mechanical Engineering, 2008. 44(04):47. doi:10.3901/JME.2008.04.047.

Lu, Y., Wang, P., Hou, Z., Hu, B., Sui, C., and Han, J. Kinetostatic analysis of a novel 6DoF 3UPS parallel manipulator with multi-fingers. Mechanism and Machine Theory, 2014. 78:36-50. doi:10.1016/j.mechmachtheory.2014.02.017.

Lyzell, C. and Hovland, G. Verification of the dynamics of the 5-DOF gantry-tau parallel kinematic machine. In Iasted International Conference on Robotics and Applications. pages 445-450, 2007.

Merlet, J. P. Parallel Robots. Kluwer Academic Publishers, The Netherlands, 2002.

Pashkevich, A., Chablat, D., and Wenger, P. Stiffness analysis of overconstrained parallel manipulators. Mechanism and Machine Theory, 2009. 44(5):966982. doi:10.1016/j.mechmachtheory.2008.05.017.

Pisla, D., Gherman, B., Vaida, C., Suciu, M., and Plitea, N. An active hybrid parallel robot for minimally invasive surgery. Robotics and Computerintegrated Manufacturing, 2013. 29(4):203-221. doi:10.1016/j.rcim.2012.12.004.

Sokolov, A. and Xirouchakis, P. Dynamics analysis of a 3-DOF parallel manipulator with RCPCS joint structure. Mechanism and Machine Theory, 2007. 42(5):541-557. doi:10.1016/j. mechmachtheory. 2006.05.004.

Vaida, C., Pisla, D., Plitea, N., Gherman, B., Gyurka, B., Graur, F., and Vlad, L. Development of a voice controlled surgical robot. 2010. pages 567-574. doi:10.1007/978-90-481-9689-0_65. 
$\mathrm{Wu}$, G. and Bai, S. Design analysis and dynamic modeling of a high-speed 3T1R parallel robot for pick-and-place application. International Journal of Mechanisms and Robotic Systems, 2016. 3(2/3):237. doi:10.1504/IJMRS.2016.10001895.

Wu, G., Bai, S., and Kepler, J. Stiffness characterization of a 3 -PPR planar parallel manipulator with actuation compliance. ARCHIVE Proceedings of the Institution of Mechanical Engineers Part C Journal of Mechanical Engineering Science 1989-1996 (vols 203-210), 2015a. 229(12). doi:10.1177/0954406214557341.

Wu, G., Caro, S., Bai, S., and Kepler, J. Dynamic modeling and design optimization of a $3-$ DOF spherical parallel manipulator. Robotics and Autonomous Systems, 2014. 62(10):1377 - 1386. doi:10.1016/j.robot.2014.06.006.
Wu, J., Wang, D., and Wang, L. A control strategy of a two degrees-of-freedom heavy duty parallel manipulator. Journal of Dynamic Systems Measurement and Control, 2015b. 137(6):061007. doi:10.1115/1.4029244.

Zhang, D., Bi, Z., and Li, B. Design and kinetostatic analysis of a new parallel manipulator. Robotics and Computer Integrated Manufacturing, 2009. 25(4):782-791. doi:10.1016/j.rcim.2008.10.002.

Zhao, Yongjie, Gao, and Feng. Inverse dynamics of the 6-dof out-parallel manipulator by means of the principle of virtual work. Robotica, 2009. 27(2):259268. doi:10.1017/S0263574708004657. 